hep-th/0105046

KCL-MTH-01-10

\title{
Fusion rules and logarithmic representations of a WZW model at fractional level
}

\author{
Matthias R. Gaberdiel ${ }^{\star}$ \\ Department of Mathematics, King's College London \\ Strand, London WC2R 2LS, U.K.
}

\begin{abstract}
The fusion products of admissible representations of the $s u(2)$ WZW model at the fractional level $k=-4 / 3$ are analysed. It is found that some fusion products define representations for which the spectrum of $L_{0}$ is not bounded from below. Furthermore, the fusion products generate representations that are not completely reducible and for which the action of $L_{0}$ is not diagonalisable. The complete set of representations that is closed under fusion is identified, and the corresponding fusion rules are derived.
\end{abstract}

$05 / 2001$

* e-mail: mrg@mth.kcl.ac.uk 


\section{Introduction}

One of the best understood conformal field theories is the WZW model that can be defined for any (simple compact) group [1]. If the so-called level is chosen to be a positive integer, the theory is unitary and rational, and in fact these models are the paradigm for rational conformal field theories. The fusion rules are well known [2, 3, [4], and they can be obtained, via the Verlinde formula [5], from the modular transformation properties of the characters.

From a Lagrangian point of view, the model is only well defined if the level is integer, but the corresponding vertex operator algebra (or the meromorphic conformal field theory in the sense of [6]) can also be constructed even if this is not the case. Furthermore, it was realised some time ago that there exists a preferred set of admissible (fractional) levels for which the characters corresponding to the 'admissible' representations have simple modular properties [7]. This suggests that these admissible level WZW models define 'almost' rational conformal field theories. It is therefore interesting and important to study these theories in order to understand to which extent results valid for rational conformal field theories may also apply to more general conformal field theories.

The fusion rules of WZW models at admissible fractional level have been studied quite extensively over the years. In particular, the simplest case of $s u(2)$ at fractional level has been analysed in detail [8, 9, 10, 11, 12, 13, 14, 15, 16, 17] (for a good review about the various results see in particular [17]). All of these fusion rule calculations essentially determine the possible couplings of three representations. More precisely, given two representations, the calculations determine whether a given third representation can be contained in the fusion product of the former two.

Two different sets of 'fusion rules' have been proposed in the literature: the fusion rules of Bernard and Felder [8] whose calculations have been reproduced in [11, 14], and the fusion rules of Awata and Yamada [10] whose results have been recovered in [12, 13, 15, 16, 17]. The two calculations differ essentially by what class of representations is considered: in Bernard \& Felder only admissible representations that are highest weight with respect to the whole affine algebra (and a fixed choice of a Borel subalgebra) are considered, while in Awata \& Yamada also representations that are highest weight with respect to an arbitrary Borel subalgebra were analysed. As a consequence, the fusion rules of Awata \& Yamada 'contain' the fusion rules of Bernard \& Felder. 
In deriving the 'fusion rules' from these calculations, it is always assumed implicitly that the actual fusion product is a direct sum of representations of the kind that are considered. (This is to say, there are no additional fusion channels that one has overlooked by restricting oneself to the class of representations in question.) In particular, in both approaches it has been assumed that the fusion rules 'close' on (conformal) highest weight representations (since these are the only representations that were considered). However, as we shall explain in quite some detail, this is not true in general. In fact, the fusion product of two highest weight representations (with respect to the affine algebra) contains sometimes a representation whose $L_{0}$ spectrum is not bounded from below. ${ }^{\dagger}$ As a consequence it is not really surprising that the fusion rules described above are somewhat incomplete.

In order to be able to analyse the fusion product without assuming that it defines a (conformal) highest weight representation, we use the description of fusion that was introduced in [18,19,20,21]. Refining techniques that were developed in [21,22] we define a nested set of quotient spaces of the fusion product that allows us to uncover, step by step, more and more of the structure of the fusion product. While this approach is necessarily incomplete (since we are not able to calculate all such quotient spaces) it is sufficient to prove that the fusion product is sometimes not a direct sum of (conformal) highest weight representations. It is also sufficient to show that some of the representations we encounter are not completely decomposable; in fact, we shall find two indecomposable representations both of which have the property that $L_{0}$ is not diagonalisable. (Representations with this property are often called 'logarithmic' representations since their correlation functions have logarithmic branch cuts [23]. For some background material on this class of representations see also [24,22,25,26, 27,28]. For WZW models at level $k=0$ logarithmic representations have been discovered before in [29,30]; however these models are somewhat pathological (at $k=0$ the vacuum representation is trivial), and the relevant logarithmic representations are quite different from what will be analysed here.) In both cases the fact that $L_{0}$ is not diagonalisable is not visible when restricting to the highest weight space only. (In this respect, these representations are similar to the logarithmic representation $\mathcal{R}_{1}$ of [31].) It is therefore not surprising that these logarithmic representations were overlooked before.

$\dagger$ The representation has, however, the property that $V_{n}(\psi) \chi=0$ for $n \geq N$ (where $N$ depends on both $\psi$ and $\chi$ ); this is sufficient to guarantee that the corresponding correlation functions do not have essential singularities. 
On the other hand, where our calculations can be compared with the above calculations, they reproduce the corresponding results.

One important insight that allows us to describe the fusion rules fairly compactly is the observation that the fusion rules are symmetric under the twist symmetry that originates from the outer automorphism of the current algebra ${ }^{\ddagger}$. While we cannot prove that the actual fusion rules respect this symmetry, we give very strong circumstantial evidence for this claim. If this is indeed true (as we conjecture) then it is immediate that the fusion product of certain highest weight representations must contain representations that are not (conformal) highest weight representations. Furthermore, this symmetry allows us to group together all representations that are related in this fashion. In this way we can give compact formulae for the fusion rules (under one natural assumption that we discuss in section 8). In particular, as was the case in [31], the fusion rules close on some smaller set of representations (that contains the two indecomposable representations together with one irreducible representation, as well as their images under the twist symmetry), and we find associative fusion rules for these three representations. These fusion rules, however, bear no resemblance to the fusion rules of either Bernard \& Felder or Awata \& Yamada, since the three representations are quite different from those in either [8] or [10]. The corresponding $S$-matrix (that diagonalises the fusion rules) is also different from the $S$ matrix of Kac \& Wakimoto [7]; again, this is not surprising since the representations (and characters) are not simply the 'admissible' representations of [7].*

The paper is organised as follows. We fix our notation and describe the twist symmetry in section 2. In section 3 we analyse which (conformal) highest weight representations of the affine algebra are in fact representations of the conformal field theory (i.e. representations of the vertex operator algebra). In section 4 we describe the algorithm for the analysis of the fusion rules in some generality, and we apply it in section 5, 6 and 7 to the case at hand. In section 8 we derive the full set of fusion rules (using the associativity of the fusion product), and section 9 contains some conclusions. We have included two appendices where some calculations are spelled out in some more detail.

$\ddagger$ At integer level this symmetry gives rise to the so-called 'simple current' automorphism of the fusion rules.

* The correct interpretation for the fusion rules that correspond to the $S$-matrix of Kac \& Wakimoto was given in [32]: as we shall explain in more detail in section 8, the corresponding fusion rules agree precisely with (a subset of) our fusion rules, and indeed support the conjecture that the fusion rules have the aforementioned twist symmetry. 


\section{Notation and basic facts}

In this paper we shall consider the WZW model corresponding to $s u(2)$ at level $k$. The chiral algebra of this conformal field theory contains the affine algebra $\hat{s u}(2)$, whose modes satisfy the commutation relations

$$
\begin{aligned}
{\left[J_{m}^{+}, J_{n}^{-}\right] } & =2 J_{m+n}^{3}+k m \delta_{m,-n} \\
{\left[J_{m}^{3}, J_{n}^{ \pm}\right] } & = \pm J_{m+n}^{ \pm} \\
{\left[J_{m}^{3}, J_{n}^{3}\right] } & =\frac{k}{2} m \delta_{m,-n}
\end{aligned}
$$

By virtue of the Sugawara construction, we can define Virasoro generators as bilinears in the currents $J$; these Virasoro modes satisfy the commutation relations of the Virasoro algebra

$$
\left[L_{m}, L_{n}\right]=(m-n) L_{m+n}+\frac{c}{12} m\left(m^{2}-1\right) \delta_{m,-n},
$$

where $c$ is given in terms of the level $k$ as

$$
c=\frac{3 k}{(k+2)} \text {. }
$$

We shall mainly consider the case $k=-4 / 3$ in this paper; for this value of $k$ we have $c=-6$.

The zero modes in (2.1) satisfy the commutation relations of $s u(2)$, whose Casimir operator we denote by

$$
C=\frac{1}{2}\left(J_{0}^{+} J_{0}^{-}+J_{0}^{-} J_{0}^{+}\right)+J_{0}^{3} J_{0}^{3} .
$$

Using the commutation relations (2.1) we then have

$$
\begin{aligned}
& J_{0}^{+} J_{0}^{-}=C+J_{0}^{3}-J_{0}^{3} J_{0}^{3} \\
& J_{0}^{-} J_{0}^{+}=C-J_{0}^{3}-J_{0}^{3} J_{0}^{3} .
\end{aligned}
$$

We shall often be interested in what we shall call (conformal) highest weight representations $^{\dagger}$; these representations have the property that they are generated by the action of the currents $J_{m}^{a}$ with $m \leq 0$ from a (conformal) highest weight state $\psi$, i.e. a state satisfying

$$
J_{m}^{a} \psi=0 \quad \text { for } m>0
$$

$\dagger$ Representations with this property are often simply referred to as 'highest weight representations'. We have included the qualifier '(conformal)' in order to distinguish these representations from the highest weight representations of the affine algebra (which have the additional property that the positive roots of the zero mode algebra also annihilate $\psi$ ). 
The (conformal) highest weight states in the representation generated from $\psi$ form a representation of the zero mode algebra; if this representation is irreducible, the Casimir operator $C$ takes a specific value, $C_{\psi}$, and the conformal weight of $\psi, h_{\psi}$, is given by

$$
h_{\psi}=\frac{C_{\psi}}{(k+2)} .
$$

In the following we shall make use of the fact that the affine algebra has an automorphism defined by

$$
\begin{aligned}
& \pi_{s}\left(J_{m}^{ \pm}\right)=J_{m \mp s}^{ \pm} \\
& \pi_{s}\left(J_{m}^{3}\right)=J_{m}^{3}-\frac{k}{2} s \delta_{m, 0},
\end{aligned}
$$

where $s \in \mathbb{Z}$. The induced action on the Virasoro generators is given by

$$
\pi_{s}\left(L_{m}\right)=L_{m}-s J_{m}^{3}+\frac{1}{4} k s^{2} \delta_{m, 0} .
$$

If $s$ is even, the automorphism is inner in the sense that it can be obtained by the adjoint action of an element in the loop group of $S U(2)$; on the other hand, if $s$ is odd, the automorphism can be obtained by the adjoint action of a loop in $S O(3)$ that does not define an element in the loop group of $S U(2)$ [33,34.

For positive integer $k$, the integrable positive energy representations are characterised by the property that the highest weight states transform in a representation with Casimir $C=C(j)=j(j+1)$, where $j=0, \frac{1}{2}, \ldots, \frac{k}{2}$. In this case, the induced action of the automorphism $\pi_{1}$ on the highest weight representations is given by

$$
\pi_{1}: j \mapsto \frac{k}{2}-j
$$

In particular, $\pi_{s}$ with $s$ even maps each integrable positive energy representations into itself; this simply reflects the fact that every such representation gives rise to a representation of the full loop group, and that the automorphism for $s$ even is inner (in the sense described above).

Furthermore, at least for the case of positive integer $k$ where the fusion rules are well known [2], $\pi_{s}$ respects the fusion rules in the sense that

$$
\left(\pi_{s}\left(\mathcal{H}_{1}\right) \otimes \pi_{t}\left(\mathcal{H}_{2}\right)\right)_{\mathrm{f}}=\pi_{s+t}\left(\left(\mathcal{H}_{1} \otimes \mathcal{H}_{2}\right)_{\mathrm{f}}\right)
$$

This seems to be quite a general property of 'twist'-symmetries such as (2.8) (see for example [35] for another example of this type for the case of the $\mathcal{N}=2$ algebras); we shall therefore assume in the following that the fusion rules also satisfy this property in our case. In any case, this is consistent with what we shall find. 


\section{The set of allowed representations}

At $k=-4 / 3$, the vacuum representation has one (independent) null-vector

$$
\mathcal{N}=\left(J_{-3}^{3}+\frac{3}{2} J_{-2}^{+} J_{-1}^{-}-\frac{3}{2} J_{-1}^{+} J_{-2}^{-}+\frac{9}{2} J_{-1}^{3} J_{-1}^{+} J_{-1}^{-}+\frac{9}{2} J_{-1}^{3} J_{-1}^{3} J_{-1}^{3}-\frac{9}{2} J_{-2}^{3} J_{-1}^{3}\right)|0\rangle .
$$

The presence of a null-vector in the vacuum representation usually implies that only a subset of the representations of the affine algebra actually define representations of the meromorphic conformal field theory. In order to determine the relevant set of representations, one could determine Zhu's algebra [36] (whose representations are in one-to-one correspondence with the representations of the meromorphic conformal field theory). In practice, however, this is quite complicated since Zhu's algebra does not have a simple grading. Alternatively, we shall therefore use an approach that is commonly taken in the physics literature [37] (and that is believed to be equivalent to the determination of Zhu's algebra): we shall analyse the constraint that comes from the condition that $V_{0}(\mathcal{N}) \psi=0$, where $\psi$ is an arbitrary state in the representation space from which the whole representation is generated by the action of the modes, and $V_{n}(\phi)$ is the $n$-th mode of the vertex operator corresponding to the state $\phi$ in the vacuum representation,

$$
V(\phi, z)=\sum_{n \in \mathbb{Z}} V_{n}(\phi) z^{-n-h_{\phi}}
$$

(Here $h_{\phi}$ is the conformal weight of $\phi$.) If the representation is a (conformal) highest weight representation, it is convenient to evaluate this constraint for one of the highest weight states.

The complete expression for $V_{0}(\mathcal{N})$ is quite complicated, but if we restrict our attention to the case when $V_{0}(\mathcal{N})$ acts on a highest weight state, the formula simplifies quite significantly. In this case we find (using techniques described for example in [38])

$$
\begin{aligned}
V_{0}(\mathcal{N}) \psi & =\left[\frac{9}{2}\left(J_{0}^{-} J_{0}^{+} J_{0}^{3}+J_{0}^{3} J_{0}^{3} J_{0}^{3}+J_{0}^{3} J_{0}^{3}\right)+J_{0}^{3}\right] \psi \\
& =\left[\left(\frac{9}{2} C+1\right) J_{0}^{3}\right] \psi,
\end{aligned}
$$

where $C$ is the Casimir operator (2.4) and $\psi$ is an arbitrary (conformal) highest weight state. Since we have to have that $V_{0}(\mathcal{N}) \psi=0$ for every highest weight state in a given highest weight representation, it follows that either the highest weight representation is 
the trivial (vacuum) representation, or it has to have Casimir equal to $-2 / 9$. The vacuum representation is both highest and lowest weight with respect to the $s u(2)$ zero mode algebra, and among the representations with $C=-2 / 9$, there are four representations that are either highest or lowest weight with respect to this $s u(2)$. Let us denote by $D_{j}^{+}$ the highest weight representation that is generated from a state $|j\rangle$ satisfying

$$
\begin{array}{rlrl}
D_{j}^{+}: & J_{0}^{+}|j\rangle & =0 \\
J_{0}^{3}|j\rangle & =j|j\rangle,
\end{array}
$$

and by $D_{j}^{-}$the lowest weight representation that is generated from a state $|j\rangle$ satisfying

$$
\begin{array}{rlrl}
D_{j}^{-}: & J_{0}^{-}|j\rangle & =0 \\
J_{0}^{3}|j\rangle & =j|j\rangle .
\end{array}
$$

The value of the Casimir for these representations is given by

$$
C\left(D_{j}^{+}\right)=j(j+1), \quad C\left(D_{j}^{-}\right)=j(j-1) .
$$

Thus these representations have Casimir equal to $-2 / 9$ in the following four cases

$$
D_{-\frac{2}{3}}^{+}, \quad D_{-\frac{1}{3}}^{+}, \quad D_{\frac{2}{3}}^{-}, \quad D_{\frac{1}{3}}^{-}
$$

The two highest weight representations in (3.7) are precisely the 'admissible' representations of Kac and Wakimoto whose characters were found to have simple modular transformation properties [7]. The fact that these admissible representations are indeed representations of the meromorphic conformal field theory was shown, using slightly different methods, in [14].

However, it is clear that there exist also representations with $C=-2 / 9$ that are neither highest nor lowest weight with respect to the $s u(2)$ zero modes. As we shall see, one of them will play an important role for the description of the fusion rules. This $s u(2)$ representation (which we shall denote by $E$ in the following) consists of the states $|m\rangle$, $m \in \mathbb{Z}$, for which

$$
\begin{aligned}
J_{0}^{3}|m\rangle & =m|m\rangle \\
J_{0}^{+}|m\rangle & =|m+1\rangle \\
J_{0}^{-}|m\rangle & =\left(-\frac{2}{9}-m(m-1)\right)|m-1\rangle .
\end{aligned}
$$

These conditions characterise the representation $E$ uniquely. 
Obviously, these are not the only allowed representations: for every $t \in[0,1)$ with $t \neq \frac{1}{3}, \frac{2}{3}$, there exists a representation $E_{t}$ that consists of the states $|m\rangle$ with $m-t \in \mathbb{Z}$ for which the action of $s u(2)$ is defined by (3.8). In particular, this implies that the theory is not rational (as had been previously shown in [39]).

On every (conformal) highest weight state, the action of $L_{0}$ is proportional to the Casimir $C$. As we have seen above, in order for the highest weight state to belong to a (non-trivial) representation of the conformal field theory, the Casimir must take a definite value, $C=-2 / 9$. As a consequence, every allowed highest weight state is an eigenstate of $L_{0}$ (with eigenvalue $h=-1 / 3$ ). Thus one may be tempted to believe that the theory does not have any 'logarithmic' representations (which are characterised by the property that $L_{0}$ is not diagonalisable). Quite surprisingly, this is however not true. As we shall see, the fusion product of two highest weight representations contains a representation that is not a (conformal) highest weight representation, and for which the action of $L_{0}$ is not diagonalisable.

All of these highest weight representations have non-trivial null vectors. At grade one, the null vector is explicitly given by

$$
\mathcal{N}_{1}=\left[\left(9 m^{2}-1\right) J_{-1}^{3}+\frac{9}{4}\left(2 m+\frac{2}{3}\right) J_{-1}^{+} J_{0}^{-}+\frac{9}{4}\left(2 m-\frac{2}{3}\right) J_{-1}^{-} J_{0}^{+}\right]|m\rangle,
$$

where $|m\rangle$ has $J_{0}^{3}$ eigenvalue $m$ (and Casimir $C=-2 / 9$ ). In fact, $\mathcal{N}_{1}=V_{-1}(\mathcal{N})|m\rangle$, where $\mathcal{N}$ is the vacuum null vector (3.1). There is also a non-trivial null vector at grade two (i.e. a null-vector that is not a descendant of $\left.\mathcal{N}_{1}\right)$; it is given by $\mathcal{N}_{2}=V_{-2}(\mathcal{N})|m\rangle$, and its explicit expression is

$$
\begin{aligned}
\mathcal{N}_{2}= & \left(9 m^{2}+\frac{9}{2} m-1\right) J_{-2}^{3}+\frac{3}{2}(3 m+2) J_{-2}^{+} J_{0}^{-}+\frac{3}{2}(3 m-2) J_{-2}^{-} J_{0}^{+} \\
& \left.+\frac{27}{2} m J_{-1}^{3} J_{-1}^{3}+\frac{9}{2} m J_{-1}^{-} J_{-1}^{+}+\frac{9}{2} J_{-1}^{3} J_{-1}^{+} J_{0}^{-}+\frac{9}{2} J_{-1}^{3} J_{-1}^{-} J_{0}^{+}\right]|m\rangle .
\end{aligned}
$$

Some of the representations (3.7) are interrelated by the automorphisms $\pi_{s}$. In order to describe these relations, let us introduce the following notation. If $\rho: \mathcal{A} \rightarrow \operatorname{End}(\mathcal{H})$ describes the representation $\mathcal{H}$, then we denote by $\pi_{s}(\mathcal{H})$ the representation that is defined by $\rho \circ \pi_{s}$. With this notation we then have

$$
\begin{aligned}
\pi_{1}\left(\mathcal{H}_{0}\right) & =D_{\frac{2}{3}}^{-} \\
\pi_{-1}\left(\mathcal{H}_{0}\right) & =D_{-\frac{2}{3}}^{+} \\
\pi_{1}\left(D_{-\frac{1}{3}}^{+}\right) & =D_{\frac{1}{3}}^{-} .
\end{aligned}
$$


Here $\mathcal{H}_{0}$ denotes the vacuum representation. The first two lines imply that $\pi_{2}\left(D_{-\frac{2}{3}}^{+}\right)=$ $D_{\frac{2}{3}}^{-}$. Apart from these special cases, the application of an automorphism to any of these representations typically leads to a representation that does not have the (conformal) highest weight property. However, all representations that arise in this fashion have the property that for a given state $\psi$ in the representation and a given $\chi$ in the vacuum representation, there exists a positive integer $N$ (that depends on $\psi$ and $\chi$ ) such that

$$
V_{n}(\chi) \psi=0 \quad \text { for all } n>N .
$$

This truncation property is sufficient to guarantee that all correlation functions will only have poles (rather than essential singularities).

\section{The fusion algorithm}

Before we begin to describe our results in detail, let us briefly explain some of the techniques that we shall be using in the following. As was explained in [19,20,21] fusion can be defined in terms of a ring-like tensor product: given two representations of the chiral algebra, $\mathcal{H}_{1}$ and $\mathcal{H}_{2}$, the tensor product $\left(\mathcal{H}_{1} \otimes \mathcal{H}_{2}\right)$ carries two natural actions of the chiral algebra (that are defined by the comultiplication formulae [18, 19,20,21]), and the fusion product $\left(\mathcal{H}_{1} \otimes \mathcal{H}_{2}\right)_{\mathrm{f}}$ is the quotient space of the tensor product where we identify these two actions. The main advantage of this description relative to most other approaches to fusion is that we do not presuppose that the fusion product has any specific properties. In most other fusion calculations one only analyses whether one of the familiar (highest weight) representations is contained in the fusion product, but here we shall analyse the fusion product itself, not just some subrepresentations it may contain. In fact, while in all cases that have been analysed so far, the fusion product of two highest weight representations is highest weight, there is no abstract reason why this has to be so, and indeed, in the present context we shall find that this is not the case. As we shall see, the fusion product of two (conformal) highest weight representations defines a representation that is not a (conformal) highest weight representation. We shall also find that the fusion product of certain (conformal) highest weight representations defines a reducible but indecomposable representation for which the action of $L_{0}$ is not diagonalisable.

In principle, one would like to describe the full space $\left(\mathcal{H}_{1} \otimes \mathcal{H}_{2}\right)_{\mathrm{f}}$ directly, but unfortunately, this is a fairly hopeless task. Instead, we shall therefore analyse a number of nested quotient spaces; these will give more and more information about the fusion product and 
will allow us to show certain properties (and to make very strongly supported conjectures for others). The most important quotient space is the space that we obtain by quotienting out all states that can be obtained by the action of the negative modes $\mathcal{A}_{-}$(i.e. the modes $V_{n}(\phi)$ with $\left.n<0\right)$ [21,

$$
\left(\mathcal{H}_{1} \otimes \mathcal{H}_{2}\right)_{\mathrm{f}}^{(0)} \equiv\left(\mathcal{H}_{1} \otimes \mathcal{H}_{2}\right)_{\mathrm{f}} / \mathcal{A}_{-}\left(\mathcal{H}_{1} \otimes \mathcal{H}_{2}\right)_{\mathrm{f}}
$$

where the action of the chiral algebra on the fusion product is defined in terms of the comultiplication formulae [18,19,20]. It is clear that this space carries a natural action of the zero modes. Furthermore, the space is naturally dual to the highest weight space of the conjugate representation, and therefore, for an irreducible highest weight representation, can be identified with the highest weight space itself; in the following we shall therefore sometimes refer to it as the 'highest weight space of the fusion product'. It can be efficiently computed using the algorithm described in [22] (see also [21]): choosing suitable insertion points for the two representations $\left(z_{1}=1, z_{2}=0\right)$, we have the identities

$$
\begin{aligned}
& \left(\mathbb{1} \otimes J_{-n}^{a}\right) \cong-\left(J_{0}^{a} \otimes \mathbb{1}\right)+\left(\mathcal{A}_{+} \otimes \mathbb{1}\right) \\
& \left(J_{-n}^{a} \otimes \mathbb{1}\right) \cong-(-1)^{n}\left(\mathbb{1} \otimes J_{0}^{a}\right)+\left(\mathbb{1} \otimes \mathcal{A}_{+}\right)
\end{aligned}
$$

where $\mathcal{A}_{+}$is the subalgebra of positive modes and we have assumed that $n \geq 1$. Thus if we are evaluating (4.1) on (conformal) highest weight states in $\mathcal{H}_{1}$ and $\mathcal{H}_{2}$, the second terms in (4.2) vanish.

As an aside, one can use this approach to analyse the allowed representations of the conformal field theory, following an idea described in [40. To this end, we consider the fusion product of an arbitrary (conformal) highest weight representation with the vacuum representation, and analyse the conditions under which the original highest weight representation is contained in this fusion product, and therefore in (4.1). Since the vacuum representation has a null vector (3.1), we obtain a non-trivial constraint from using (4.2) repeatedly,

$$
\begin{aligned}
0 & =(\psi \otimes \mathcal{N}) \\
& \cong\left(-J_{0}^{3}+\frac{3}{2} J_{0}^{-} J_{0}^{+}-\frac{3}{2} J_{0}^{-} J_{0}^{+}-\frac{9}{2} J_{0}^{-} J_{0}^{+} J_{0}^{3}-\frac{9}{2} J_{0}^{3} J_{0}^{3} J_{0}^{3}-\frac{9}{2} J_{0}^{3} J_{0}^{3}\right) \psi \otimes|0\rangle \\
& \cong-\left(\left[\left(1+\frac{9}{2} C\right) J_{0}^{3}\right] \psi \otimes|0\rangle\right)
\end{aligned}
$$


where $C$ is the Casimir operator (2.4) and $\psi$ is an arbitrary highest weight vector. This reproduces $(3.3)$.

As we shall see in the next section, the knowledge of (4.1) is sometimes not sufficient to characterise the fusion product uniquely. When appropriate we shall therefore also consider a slightly larger quotient space (that therefore captures slightly more information about the fusion product). In particular, we shall consider the quotient space

$$
\left(\mathcal{H}_{1} \otimes \mathcal{H}_{2}\right)_{\mathrm{f}}^{(+1)} \equiv\left(\mathcal{H}_{1} \otimes \mathcal{H}_{2}\right)_{\mathrm{f}} / \mathcal{A}_{-}^{+1}\left(\mathcal{H}_{1} \otimes \mathcal{H}_{2}\right)_{\mathrm{f}}
$$

where $\mathcal{A}_{-}^{+1}$ is the algebra that is spanned by the modes

$$
\begin{array}{lll}
\mathcal{A}_{-}^{+1}: & J_{-n}^{+} & \text {with } n \geq 2 \\
& J_{-n}^{3} & \text { with } n \geq 1 \\
& J_{-n}^{-} & \text {with } n \geq 1 .
\end{array}
$$

It is easy to see that $\mathcal{A}_{-}^{+1}$ closes among these modes. It is also clear that (4.1) is a natural quotient space of (4.4); in this sense the two quotient spaces are nested. Finally, it is worth mentioning that $L_{0}, J_{0}^{3}$ and $J_{0}^{-}$(but not $J_{0}^{+}$) act in a well-defined manner on this quotient space.

In order to determine (4.4) we can use essentially the same algorithm as before for (4.1). In fact, (4.2) still holds provided that $n \geq 1$ for $a=3,-$ and $n \geq 2$ for $a=+$. In addition we have the identity

$$
\left(J_{-1}^{+} \otimes \mathbb{1}\right) \cong\left(\mathbb{1} \otimes J_{-1}^{+}\right)+\sum_{m=0}^{\infty}(-1)^{m}\left(J_{m}^{+} \otimes \mathbb{1}\right)+\sum_{l=0}^{\infty}\left(\mathbb{1} \otimes J_{l}^{+}\right)
$$

The last identity comes from the fact that, on the fusion product, $\Delta_{1,0}\left(J_{-1}^{+}\right)$and $\widetilde{\Delta}_{0,-1}\left(J_{-1}^{+}\right)$only differ by states that lie in the quotient space of (4.4). (Here we have used the notation of [19,20].)

Ideally one would like to determine yet bigger quotient spaces. However, the complexity of the calculation increases very quickly, and the above is essentially the limit of what can be calculated feasibly by hand. An implementation of the calculation on a computer is not straightforward since all highest weight spaces are infinite dimensional, and thus the computer algorithm used in 22] cannot be applied directly. At any rate, the above quotient spaces are already sufficient to show that the fusion product of certain highest weight representations contains a logarithmic representation. 


\section{The fusion rules}

We are now in the position to work out the various fusion products. Let us begin by determining the 'highest weight space' (4.1) of the fusion product of $D_{-\frac{2}{3}}^{+}$with an arbitrary (conformal) highest weight representation $\mathcal{H}$. First of all, we can use (4.2) repeatedly to reduce any state in the fusion product (up to states in the quotient space) to a sum of products of highest weight states. Next we want to obtain the constraints that follow from the existence of the null vectors in $D_{-\frac{2}{3}}^{+}$. To this end, it is useful to observe that for $m=-\frac{2}{3}$,

$$
\mathcal{N}_{-\frac{2}{3}}^{+}=J_{0}^{+} \mathcal{N}_{1}=-J_{-1}^{+}\left|-\frac{2}{3}\right\rangle
$$

is also a null-vector in $D_{-\frac{2}{3}}^{+}$(as one can easily check directly). Using (4.2) together with this null-vector we get the condition

$$
\begin{aligned}
0 & =\left(\psi \otimes \mathcal{N}_{-\frac{2}{3}}^{+}\right) \\
& \cong\left(J_{0}^{+} \psi \otimes\left|-\frac{2}{3}\right\rangle\right),
\end{aligned}
$$

where $\psi \in \mathcal{H}^{(0)}$ is an arbitrary (conformal) highest weight state in $\mathcal{H}$. (Here and in the following we shall denote by $\mathcal{H}^{(0)}$ the subspace of (conformal) highest weight states of $\mathcal{H}$.) If $\mathcal{H}=D_{-\frac{1}{3}}^{+}$or $\mathcal{H}=D_{-\frac{2}{3}}^{+}$, then every $\phi \in \mathcal{H}^{(0)}$ can be written as $\phi=J_{0}^{+} \psi$ for some $\psi \in \mathcal{H}^{(0)}$. Thus we find that for every $\phi \in \mathcal{H}^{(0)},(\phi \otimes|-2 / 3\rangle)$ lies in the quotient by which we divide to obtain the highest weight subspace of the fusion product. Furthermore, using recursively the relation

$$
0 \cong J_{0}^{-}\left(\phi \otimes\left|-\frac{2}{3}\right\rangle\right)=\left(J_{0}^{-} \phi \otimes\left|-\frac{2}{3}\right\rangle\right)+\left(\phi \otimes J_{0}^{-}\left|-\frac{2}{3}\right\rangle\right),
$$

we can show that all states in the tensor product of the highest weight space of $D_{-\frac{2}{3}}^{+}$and $\mathcal{H}^{(0)}$ are in the quotient space by which we divide. Thus we conclude that the fusion product of $D_{-\frac{2}{3}}^{+}$with $D_{-\frac{2}{3}}^{+}$or $D_{-\frac{1}{3}}^{+}$does not contain any (conformal) highest weight states,

$$
\left(D_{-\frac{2}{3}}^{+} \otimes D_{-\frac{2}{3}}^{+}\right)_{\mathrm{f}}^{(0)}=\left(D_{-\frac{2}{3}}^{+} \otimes D_{-\frac{1}{3}}^{+}\right)_{\mathrm{f}}^{(0)}=0 .
$$

A similar conclusion was also reached in [32]. This is in fact in agreement with the symmetry (2.11) since $D_{-\frac{2}{3}}^{+}=\pi_{-1}\left(\mathcal{H}_{0}\right)$, and we therefore expect that

$$
\begin{aligned}
& \left(D_{-\frac{2}{3}}^{+} \otimes D_{-\frac{2}{3}}^{+}\right)_{\mathrm{f}}=\pi_{-1}\left(D_{-\frac{2}{3}}^{+}\right) \\
& \left(D_{-\frac{2}{3}}^{+} \otimes D_{-\frac{1}{3}}^{+}\right)_{\mathrm{f}}=\pi_{-1}\left(D_{-\frac{1}{3}}^{+}\right) .
\end{aligned}
$$


Indeed, it is easy to see that the right hand side of (5.5) does not define a (conformal) highest weight representation.

We can test (5.5) further by determining the quotient space (4.4) that captures more than just the highest weight states. For the case when both representations are $D_{-\frac{2}{3}}^{+}$we have done this calculation, and we have found that ${ }^{\ddagger}$

$$
\left(D_{-\frac{2}{3}}^{+} \otimes D_{-\frac{2}{3}}^{+}\right)_{\mathrm{f}}^{(+1)}=\left\{(\hat{m}, \hat{m}): \hat{m}=-\frac{4}{3},-\frac{7}{3}, \ldots\right\},
$$

where the first entry of $(\hat{m}, \hat{m})$ refers to its $J_{0}^{3}$ eigenvalue, and the second to its $L_{0}$ eigenvalue,

$$
\begin{aligned}
& J_{0}^{3}(\hat{m}, \hat{m})=\hat{m}(\hat{m}, \hat{m}) \\
& L_{0}(\hat{m}, \hat{m})=\hat{m}(\hat{m}, \hat{m}) .
\end{aligned}
$$

This agrees with the spectrum of $J_{0}^{3}$ and $L_{0}$ on the quotient space of $\pi_{-1}\left(D_{-\frac{2}{3}}^{+}\right)$where we divide out the image of $\mathcal{A}_{-}^{+1}$ : the states that survive in this quotient space are the image (under $\pi_{-1}$ ) of the original highest weight states in $D_{-\frac{2}{3}}^{+}$. Using (2.8) and (2.9) the spectrum of these states is then precisely described by (5.6) and (5.7).

If $\mathcal{H}=D_{\frac{2}{3}}^{-}$, then the first part of the argument is similar, except now there is one state in the highest weight space of $D_{\frac{2}{3}}^{-}$that cannot be written as $J_{0}^{+} \phi$ : this is the state $|2 / 3\rangle$. Using (5.3) as before this allows us then to show that all tensor products of highest weight states for which the total $J_{0}^{3}$ eigenvalue is bigger than zero lie in the quotient by which we divide. (Here the total $J_{0}^{3}$ eigenvalue is the sum of the two $J_{0}^{3}$ eigenvalues.) We can similarly use the null-vector $\mathcal{N}_{1}$ of $D_{\frac{2}{3}}^{-}$(or rather, as before in (5.1), the null vector that is obtained from $\mathcal{N}_{1}$ by the action of $J_{0}^{-}$) to deduce that the same holds for those tensor products whose total $J_{0}^{3}$ eigenvalue is less than zero. This leaves us with the states in the tensor product for which the total $J_{0}^{3}$ eigenvalue is zero: these are the states of the form

$$
\left(J_{0}^{+}\right)^{l}\left|\frac{2}{3}\right\rangle \otimes\left(J_{0}^{-}\right)^{l}\left|-\frac{2}{3}\right\rangle,
$$

where $l=0,1, \ldots$.. By considering

$$
0=\left(J_{0}^{-}\right)^{m+1}\left(\left(J_{0}^{+}\right)^{m}\left|\frac{2}{3}\right\rangle \otimes J_{-1}^{+}\left|-\frac{2}{3}\right\rangle\right)
$$

$\ddagger$ Strictly speaking, our calculation only allows us to derive an upper bound on the size of the quotient space. However, we have used all available null vector relations, and we are therefore confident that this bound is actually saturated. This comment applies equally to all other calculations of quotient spaces in this paper. 
and using the algorithm above, we obtain a recursion relation for the state with $l=l_{0}$ in (5.8) in terms of states with $l=0,1, \ldots, l_{0}-1$. Thus the highest weight space is actually one-dimensional, and it consists of a state of $J_{0}^{3}$ eigenvalue zero. This suggests that we have

$$
\left(D_{-\frac{2}{3}}^{+} \otimes D_{\frac{2}{3}}^{-}\right)_{\mathrm{f}}=\mathcal{H}_{0}
$$

We have also checked that the null-vectors $\mathcal{N}_{2}$ for both representations do not give rise to any additional constraints.

Finally we find, using similar arguments,

$$
\begin{aligned}
& \left(D_{-\frac{2}{3}}^{+} \otimes D_{\frac{1}{3}}^{-}\right)_{\mathrm{f}}=D_{-\frac{1}{3}}^{+} \\
& \left(D_{\frac{2}{3}}^{-} \otimes D_{-\frac{1}{3}}^{+}\right)_{\mathrm{f}}=D_{\frac{1}{3}}^{-} .
\end{aligned}
$$

All of these results are in agreement with (2.11) and (3.11), and in fact could have been deduced from this symmetry.

The only fusion products involving the representations (3.7) that are not determined in terms of this symmetry are the fusion products involving $D_{-\frac{1}{3}}^{+}$and $D_{\frac{1}{3}}^{-}$. Using the algorithm described above we have calculated the highest weight space

$$
\left(D_{-\frac{1}{3}}^{+} \otimes D_{\frac{1}{3}}^{-}\right)_{\mathrm{f}}^{(0)}=\{|0\rangle\} \oplus E
$$

where $|0\rangle$ is a state with $J_{0}^{3}$ and $L_{0}$ eigenvalue zero, and $E$ is the representation that was discussed in section 3. (This result agrees with the $x=\infty$ limit (that corresponds to a specific choice for the Borel subalgebra for one of the three representations) described in [17]: the representation $E$ does not appear in their fusion product since $E$ is not an (affine) highest weight representation.) Furthermore, we have checked that

$$
\left(D_{-\frac{1}{3}}^{+} \otimes D_{\frac{1}{3}}^{-}\right)_{\mathrm{f}}^{(+1)}=\{(m, m): m=0,1,2, \ldots\} \bigoplus E
$$

where both the $J_{0}^{3}$ and $L_{0}$ eigenvalue of $(m, m)$ is $m$. Taking these results together this suggests that the actual fusion product is

$$
\left(D_{-\frac{1}{3}}^{+} \otimes D_{\frac{1}{3}}^{-}\right)_{\mathrm{f}}=\mathcal{H}_{0} \oplus \mathcal{H}_{E}
$$

where $\mathcal{H}_{E}$ is the representation of the affine algebra whose (conformal) highest weight space $\mathcal{H}_{E}^{(0)}$ is $E$. Indeed, since $\mathcal{H}_{E}$ has a null vector at grade one, $\mathcal{N}_{1}$, for which the coefficient 
of $J_{-1}^{+}$does not vanish (see $(\underline{3.9})$ ), only the ground states of $\mathcal{H}_{E}$ contribute in $(5.13)$; on the other hand, the first term in (5.13) corresponds to the states of the form $\left(J_{-1}^{+}\right)^{l}|0\rangle$ that survive the quotient by the image of $\mathcal{A}_{-}^{+1}$ in the vacuum representation. Using the automorphism symmetry (2.11) this result then also implies

$$
\begin{gathered}
\left(D_{-\frac{1}{3}}^{+} \otimes D_{-\frac{1}{3}}^{+}\right)_{\mathrm{f}}=D_{-\frac{2}{3}}^{+} \oplus \pi_{-1}\left(\mathcal{H}_{E}\right) \\
\left(D_{\frac{1}{3}}^{-} \otimes D_{\frac{1}{3}}^{-}\right)_{\mathrm{f}}=D_{\frac{2}{3}}^{-} \oplus \pi_{1}\left(\mathcal{H}_{E}\right) .
\end{gathered}
$$

Both of these results are again in agreement with the direct calculation of their highest weight spaces,

$$
\begin{gathered}
\left(D_{-\frac{1}{3}}^{+} \otimes D_{-\frac{1}{3}}^{+}\right)_{\mathrm{f}}^{(0)}=\left(D_{-\frac{2}{3}}^{+}\right)^{(0)} \\
\left(D_{\frac{1}{3}}^{-} \otimes D_{\frac{1}{3}}^{-}\right)_{\mathrm{f}}^{(0)}=\left(D_{\frac{2}{3}}^{-}\right)^{(0)},
\end{gathered}
$$

since the highest weight space of $\pi_{ \pm 1}\left(\mathcal{H}_{E}\right)$ is empty. Again, these calculations are in agreement with the $x=0$ limit of the calculation described in [17].

\section{The fusion of $\mathcal{H}_{E}$ with $D_{\mp \frac{1}{3}}^{ \pm}$}

So far we have described all fusion products involving the original representations in (3.7). We have found that the fusion rules do not close among these representations (and their images under $\pi_{s}$ ) alone, but rather that we generate the representation $\mathcal{H}_{E}$ (as well as its images under $\pi_{s}$ ). In order to describe the full fusion ring we therefore need to study the fusion of $\mathcal{H}_{E}$ with all representations in (3.7) as well as itself. First we consider the fusion of $\mathcal{H}_{E}$ with $D_{\mp \frac{2}{3}}^{ \pm}$for which the symmetry (2.11) predicts

$$
\left(D_{\mp \frac{2}{3}}^{ \pm} \otimes \mathcal{H}_{E}\right)_{\mathrm{f}}=\pi_{\mp 1}\left(\mathcal{H}_{E}\right)
$$

Again, we have checked that the highest weight spaces agree on both sides (both are empty). This leaves us with the fusion of $\mathcal{H}_{E}$ with $D_{\mp \frac{1}{3}}^{ \pm}$which we denote as

$$
\begin{aligned}
\left(D_{-\frac{1}{3}}^{+} \otimes \mathcal{H}_{E}\right)_{\mathrm{f}} & =\mathcal{R}_{-\frac{1}{3}} \\
\left(D_{\frac{1}{3}}^{-} \otimes \mathcal{H}_{E}\right)_{\mathrm{f}} & =\mathcal{R}_{\frac{1}{3}}=\pi_{1}\left(\mathcal{R}_{-\frac{1}{3}}\right)
\end{aligned}
$$

In relating the two results we have again used the symmetry (2.11). 
The analysis of $\mathcal{R}_{-\frac{1}{3}}$ is actually quite subtle, and we therefore describe it in some detail. First we determine the 'highest weight space' of $\mathcal{R}_{-\frac{1}{3}}$, i.e. the quotient of $\mathcal{R}_{-\frac{1}{3}}$ by the states that lie in the image of $\mathcal{A}_{-}$using the algorithm described above. We find that

$$
\left(\mathcal{R}_{-\frac{1}{3}}\right)^{(0)}=\left\{\left(m-\frac{1}{3},-\frac{1}{3}\right): m \in \mathbb{Z}\right\},
$$

where the action of the zero modes on $\left(m-\frac{1}{3},-\frac{1}{3}\right)$ is defined by

$$
\begin{aligned}
J_{0}^{3}\left(m-\frac{1}{3},-\frac{1}{3}\right) & =\left(m-\frac{1}{3}\right)\left(m-\frac{1}{3},-\frac{1}{3}\right) \\
L_{0}\left(m-\frac{1}{3},-\frac{1}{3}\right) & =-\frac{1}{3}\left(m-\frac{1}{3},-\frac{1}{3}\right) \\
J_{0}^{+}\left(m-\frac{1}{3},-\frac{1}{3}\right) & =\left(m+1-\frac{1}{3},-\frac{1}{3}\right) \\
J_{0}^{-}\left(m-\frac{1}{3},-\frac{1}{3}\right) & =-(m-1)\left(m-\frac{2}{3}\right)\left(m-1-\frac{1}{3},-\frac{1}{3}\right) .
\end{aligned}
$$

This representation of $s u(2)$ is reducible, but not decomposable: because of the factor of $(m-1)$ on the right hand side of the last equation, the states $\left(m-\frac{1}{3},-\frac{1}{3}\right)$ with $m \geq 1$ form an irreducible subrepresentation (that is equivalent to $\left(D_{\frac{2}{3}}^{-}\right)^{(0)}$ ), but the complementary space does not define a representation since $J_{0}^{+}\left(-\frac{1}{3},-\frac{1}{3}\right)=\left(\frac{2}{3},-\frac{1}{3}\right)$. The structure of this representation can schematically be described by

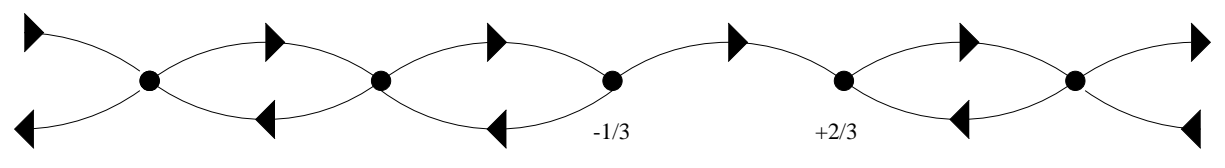

Fig. 1: The structure of $C_{-\frac{1}{3}}=\left(\mathcal{R}_{-\frac{1}{3}}\right)^{(0)}$. The arrows describe the action of $J_{0}^{ \pm}$.

We can similarly determine the 'highest weight space' of $\mathcal{R}_{\frac{1}{3}}$ and we find that

$$
\left(\mathcal{R}_{\frac{1}{3}}\right)^{(0)}=\left\{\left(\frac{1}{3}+m,-\frac{1}{3}\right): m \in \mathbb{Z}\right\}
$$

where now

$$
\begin{aligned}
J_{0}^{3}\left(m+\frac{1}{3},-\frac{1}{3}\right) & =\left(m+\frac{1}{3}\right)\left(m+\frac{1}{3},-\frac{1}{3}\right) \\
L_{0}\left(m+\frac{1}{3},-\frac{1}{3}\right) & =-\frac{1}{3}\left(m+\frac{1}{3},-\frac{1}{3}\right) \\
J_{0}^{-}\left(m+\frac{1}{3},-\frac{1}{3}\right) & =\left(m-1+\frac{1}{3},-\frac{1}{3}\right) \\
J_{0}^{+}\left(m+\frac{1}{3},-\frac{1}{3}\right) & =-(m+1)\left(m+\frac{2}{3}\right)\left(m+1+\frac{1}{3},-\frac{1}{3}\right) .
\end{aligned}
$$


Again the states $\left(m+\frac{1}{3},-\frac{1}{3}\right)$ with $m \leq-1$ form an irreducible subrepresentation (that is equivalent to $\left.\left(D_{-\frac{2}{3}}^{+}\right)^{(0)}\right)$, but the complementary space does not define a representation since $J_{0}^{-}\left(\frac{1}{3},-\frac{1}{3}\right)=\left(-\frac{2}{3},-\frac{1}{3}\right)$. Its structure is described by the 'mirror' image of that sketched in Figure 1.

Based on these results one could believe that the representations $\mathcal{R}_{\mp \frac{1}{3}}$ are simply the affine representations whose actual highest weight spaces are the indecomposable $s u(2)$ representations that are described by (6.4) and (6.6), respectively. However, this is not correct. One of the reasons why one may be suspicious about this conjecture is that the two representations $\mathcal{R}_{\mp \frac{1}{3}}$ would then not be related by $\pi_{ \pm 1}$, and thus the symmetry (2.11) would not hold any more for (6.2). In fact, if one postulates that $\mathcal{R}_{\mp \frac{1}{3}}$ are related by $\pi_{ \pm 1}$, it is easy to see that neither of the two representations can be a highest weight representation.

In order to analyse the situation further we have therefore determined the quotient space (4.4) of the first fusion product in (6.2), and we have found that

$$
\begin{aligned}
\left(\mathcal{R}_{-\frac{1}{3}}\right)^{(+1)}= & \left.\left(m-\frac{1}{3},-\frac{1}{3}\right): m \in \mathbb{Z}, m \neq 0\right\} \bigoplus\left\{\left(m-\frac{1}{3}, m-\frac{1}{3}\right): m \in \mathbb{Z}, m \neq 0\right\} \\
& \bigoplus\left\{\left(-\frac{1}{3},-\frac{1}{3}\right)_{1},\left(-\frac{1}{3},-\frac{1}{3}\right)_{2},\left(-\frac{1}{3}, \frac{2}{3}\right)\right\} .
\end{aligned}
$$

As before, the labels of the different states characterise their $J_{0}^{3}$ and $L_{0}$ eigenvalues, except for the two states $\left(-\frac{1}{3},-\frac{1}{3}\right)_{1}$ and $\left(-\frac{1}{3},-\frac{1}{3}\right)_{2}$ for which the action of $L_{0}$ is given by

$$
\begin{aligned}
& L_{0}\left(-\frac{1}{3},-\frac{1}{3}\right)_{1}=-\frac{1}{3}\left(-\frac{1}{3},-\frac{1}{3}\right)_{1}+\left(-\frac{1}{3},-\frac{1}{3}\right)_{2} \\
& L_{0}\left(-\frac{1}{3},-\frac{1}{3}\right)_{2}=-\frac{1}{3}\left(-\frac{1}{3},-\frac{1}{3}\right)_{2} .
\end{aligned}
$$

In particular, this implies that the representation $\mathcal{R}_{-\frac{1}{3}}$ is a logarithmic representation. Since this is one of the central results of this paper, we shall describe its derivation in some more detail in the appendix.

Roughly speaking, the space $\left(\mathcal{R}_{-\frac{1}{3}}\right)^{(+1)}$ consists of the states in $\left(\mathcal{R}_{-\frac{1}{3}}\right)^{(0)}-$ these are the states $\left(m-\frac{1}{3},-\frac{1}{3}\right)$ - as well as the states in $\pi_{-1}\left(\left(\mathcal{R}_{\frac{1}{3}}\right)^{(0)}\right)$ - these are the states $\left(m-\frac{1}{3}, m-\frac{1}{3}\right)$. The latter space is naturally a quotient space of $\left(\mathcal{R}_{-\frac{1}{3}}\right)^{(+1)}$ since under $\pi_{-1}$, the subspace that is generated by the negative modes becomes the space that is generated by $J_{-n}^{+}$with $n \geq 2, J_{-n}^{3}$ with $n \geq 1$ and $J_{-n}^{-}$with $n \geq 0$. The relation to 
$\left(\mathcal{R}_{\frac{1}{3}}\right)^{(0)}$ (under the map $\pi_{-1}$ ) suggests that $J_{-1}^{+}$and $J_{1}^{-}$are the 'step'-operators that move between the states $\left(m-\frac{1}{3}, m-\frac{1}{3}\right)$. Because of the structure of (6.6) we then know that

$$
J_{-1}^{+}\left(-\frac{4}{3},-\frac{4}{3}\right) \cong 0
$$

in the quotient space of $\left(\mathcal{R}_{-\frac{1}{3}}\right)^{(+1)}$ by the action of $J_{0}^{-}$. (This is the quotient space that corresponds to $\pi_{-1}\left(\left(\mathcal{R}_{\frac{1}{3}}\right)^{(0)}\right)$.) On the other hand, we can calculate the action of $J_{0}^{-}$on $\left(\mathcal{R}_{-\frac{1}{3}}\right)^{(+1)}$, and we find (for some suitable normalisation) $J_{0}^{-}\left(m-\frac{1}{3},-\frac{1}{3}\right)=\left(m-1-\frac{1}{3},-\frac{1}{3}\right)$ provided that $m \neq 1,0$. Furthermore, if $m=1$ we have $J_{0}^{-}\left(\frac{2}{3},-\frac{1}{3}\right)=\left(-\frac{1}{3},-\frac{1}{3}\right)_{2}$. This is in agreement with the description of (6.4) since, as is shown in the appendix, $\left(-\frac{1}{3},-\frac{1}{3}\right)_{2} \cong 0$ in the 'highest weight space'. This suggests that we must have (up to some constant that we can absorb into the definition of the states)

$$
J_{0}^{-}\left(\frac{2}{3},-\frac{1}{3}\right)=J_{-1}^{+}\left(-\frac{4}{3},-\frac{4}{3}\right) .
$$

Finally, we know from the analysis of the highest weight space that $J_{0}^{+}\left(-\frac{1}{3},-\frac{1}{3}\right)_{1}=$ $\left(\frac{2}{3},-\frac{1}{3}\right)$, and from the analysis of $\pi_{-1}\left(\left(\mathcal{R}_{\frac{1}{3}}\right)^{(0)}\right)$ that $J_{1}^{-}\left(-\frac{1}{3},-\frac{1}{3}\right)_{1}=\left(-\frac{4}{3},-\frac{4}{3}\right)$. Thus (6.10) implies

$$
J_{0}^{-} J_{0}^{+}\left(-\frac{1}{3},-\frac{1}{3}\right)_{1}=\frac{1}{3}\left(-\frac{1}{3},-\frac{1}{3}\right)_{2}=J_{-1}^{+} J_{1}^{-}\left(-\frac{1}{3},-\frac{1}{3}\right)_{1} .
$$

where we have made a specific prediction for the relative normalisation constants (that will be justified further below). The resulting structure is summarised in Figure 2.

On the basis of what we have determined we cannot expect to be able to derive the structure of $\mathcal{R}_{-\frac{1}{3}}$ completely; however, we can make an ansatz for its structure, and check it against the various pieces of evidence that we have accumulated. In making this ansatz we shall be guided by the principle that the representation $\mathcal{R}_{-\frac{1}{3}}$ is as well behaved as it can possibly be. For example, we know that $\left(-\frac{1}{3},-\frac{1}{3}\right)_{1}$ is not a (conformal) highest weight state (since it is not annihilated by $J_{1}^{-}$), but we can make the ansatz that it is as close to being (conformal) highest weight as possible by postulating

$$
\begin{array}{cc}
J_{n}^{+}\left(-\frac{1}{3},-\frac{1}{3}\right)_{1}=0 & \text { for } n \geq 1 \\
J_{n}^{3}\left(-\frac{1}{3},-\frac{1}{3}\right)_{1}=0 & \text { for } n \geq 1 \\
J_{n}^{-}\left(-\frac{1}{3},-\frac{1}{3}\right)_{1}=0 & \text { for } n \geq 2 \\
J_{n}^{a} J_{0}^{+}\left(-\frac{1}{3},-\frac{1}{3}\right)_{1}=0 & \text { for } n \geq 1 .
\end{array}
$$




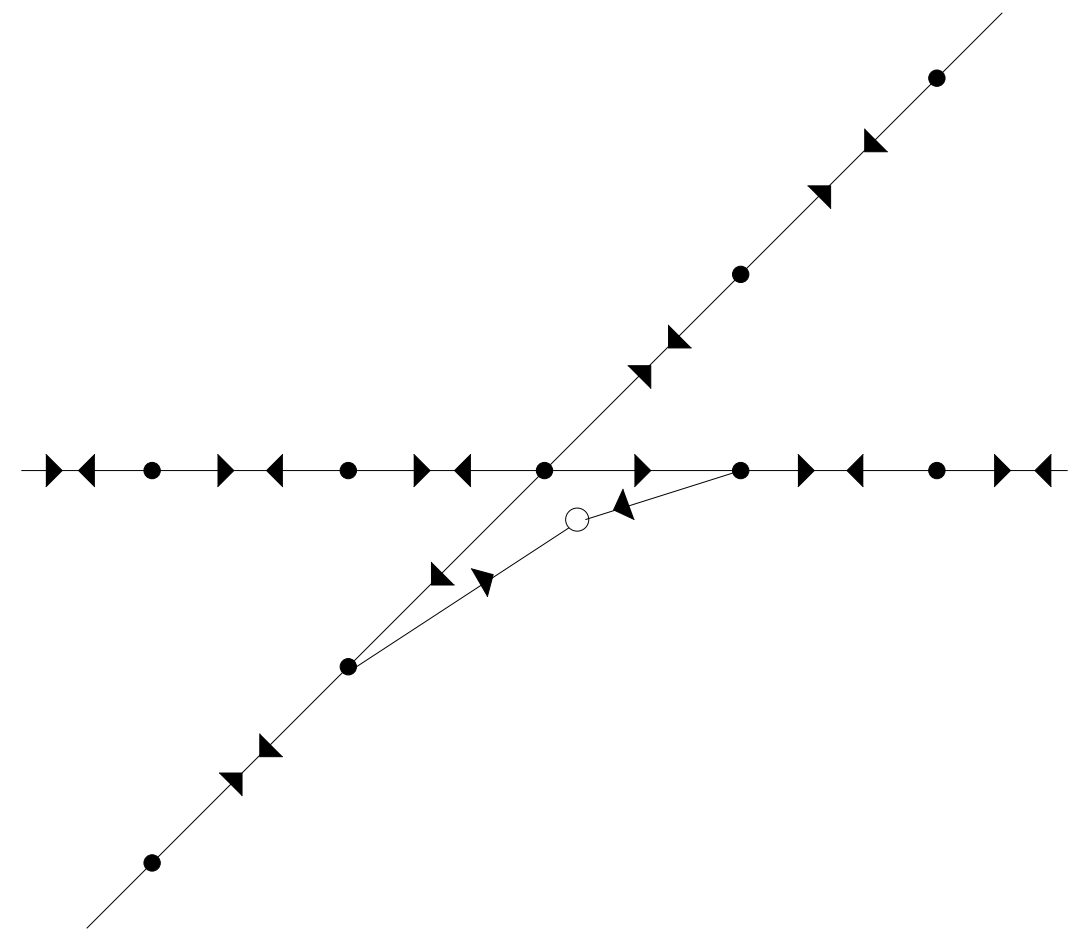

Fig. 2: The structure of the generating states of the representation $\mathcal{R}_{-\frac{1}{3}}$. Here we have arranged the circles representing the states according to their charges, with the horizontal axis corresponding to $J_{0}^{3}$, and the vertical axis to $L_{0}$. The horizontal array of circles represent the states of the form $\left(m-\frac{1}{3},-\frac{1}{3}\right)$ with $m \in \mathbb{Z}$, while the diagonal array of circles represents the states $\left(m-\frac{1}{3}, m-\frac{1}{3}\right), m \in \mathbb{Z}$. The two arrays intersect at $\left(-\frac{1}{3},-\frac{1}{3}\right)$, and the circle at this intersection corresponds to the state $\left(-\frac{1}{3},-\frac{1}{3}\right)_{1}$. The arrows indicate the action of $J_{0}^{ \pm}$(for the horizontal line) and $J_{\mp 1}^{ \pm}$(for the diagonal line). Finally, the empty circle represents the state $\left(-\frac{1}{3},-\frac{1}{3}\right)_{2}$ whose position in the charge lattice has been slightly shifted so that it does not lie on top of the other state with these charges.

As a first piece of evidence in favour of this ansatz we want to show that the resulting representation is actually an allowed representation of the conformal field theory. To this end, we want to check that $V_{0}(\mathcal{N})\left(-\frac{1}{3},-\frac{1}{3}\right)_{1}=0$ (where $\mathcal{N}$ is again the vacuum null vector), but now taking into account that $\left(-\frac{1}{3},-\frac{1}{3}\right)_{1}$ is not a highest weight. Because of this modification we now get (instead of (3.3))

$$
\begin{aligned}
0= & {\left[\frac{9}{2}\left(J_{0}^{-} J_{0}^{+} J_{0}^{3}+J_{0}^{3} J_{0}^{3} J_{0}^{3}+J_{0}^{3} J_{0}^{3}\right)+J_{0}^{3}\right.} \\
& \left.\quad+\frac{9}{2} J_{-1}^{3} J_{1}^{-} J_{0}^{+}+\frac{9}{2} J_{-1}^{+} J_{1}^{-} J_{0}^{3}+3 J_{-1}^{+} J_{1}^{-}\right]\left(-\frac{1}{3},-\frac{1}{3}\right)_{1} \\
& =\frac{3}{2}\left[J_{-1}^{+} J_{1}^{-}-J_{0}^{-} J_{0}^{+}\right]\left(-\frac{1}{3},-\frac{1}{3}\right)_{1} .
\end{aligned}
$$


Thus (6.13) follows from (6.11). We can similarly check that $V_{1}(\mathcal{N})\left(-\frac{1}{3},-\frac{1}{3}\right)_{1}=0$, but this only reproduces the same constraint. Also, $V_{L}(\mathcal{N})\left(-\frac{1}{3},-\frac{1}{3}\right)_{1}=0$ for $L \geq 2$ follows automatically from our ansatz (6.12).

Given that the representation satisfies this consistency condition, we can then construct a null-vector in $\mathcal{R}_{-\frac{1}{3}}$ by applying $V_{-1}(\mathcal{N})$ to $\left(-\frac{1}{3},-\frac{1}{3}\right)_{1}$, say (and taking care, again, of the fact that this vector only satisfies (6.12)). The null vector we obtain in this way is explicitly given as

$$
\widehat{\mathcal{N}}=\left[-3 J_{-1}^{-} J_{0}^{+}+3 J_{-2}^{+} J_{1}^{-}+\frac{9}{2} J_{-1}^{3} J_{-1}^{+} J_{1}^{-}\right]\left(-\frac{1}{3},-\frac{1}{3}\right)_{1} .
$$

By applying $J_{1}^{-}$to $\widehat{\mathcal{N}}$ we then get a non-trivial relation in $\left(\mathcal{R}_{-\frac{1}{3}}\right)^{(+1)}$, namely

$$
\begin{aligned}
0 & =\left[-3 J_{1}^{-} J_{-1}^{-} J_{0}^{+}+3 J_{1}^{-} J_{-2}^{+} J_{1}^{-}+\frac{9}{2} J_{1}^{-} J_{-1}^{3} J_{-1}^{+} J_{1}^{-}\right]\left(-\frac{1}{3},-\frac{1}{3}\right)_{1} \\
& \cong \frac{9}{2} J_{0}^{-} J_{-1}^{+} J_{1}^{-}\left(-\frac{1}{3},-\frac{1}{3}\right)_{1} \\
& =\frac{9}{2} J_{0}^{-}\left(-\frac{1}{3},-\frac{1}{3}\right)_{2} .
\end{aligned}
$$

This relation is crucial for explaining why the space of states with quantum numbers $\left(-\frac{4}{3},-\frac{1}{3}\right)$ is one-dimensional in $\left(\mathcal{R}_{-\frac{1}{3}}\right)^{(+1)}$ (rather than two-dimensional as one may have naively thought).

It is also worth mentioning that the null vector $\widehat{\mathcal{N}}$ does not involve the state $J_{-1}^{+} J_{0}^{-}\left(-\frac{1}{3},-\frac{1}{3}\right)_{1}$; this is presumably the reason why this vector is not removed from $\left(\mathcal{R}_{-\frac{1}{3}}\right)^{(+1)}$. (This is the state with quantum numbers $\left(-\frac{1}{3}, \frac{2}{3}\right)$.)

The analysis for $\mathcal{R}_{\frac{1}{3}}$ is completely analogous, and its structure is described by the 'mirror' image of Figure 2.

\section{The fusion of $\mathcal{H}_{E}$ with itself}

Finally, we need to analyse the fusion of $\mathcal{H}_{E}$ with itself. The highest weight space of this fusion product agrees precisely with the right hand side of (5.12), and one may therefore expect that the fusion of $\mathcal{H}_{E}$ with itself is precisely $\mathcal{H}_{0} \oplus \mathcal{H}_{E}$. However, given that the fusion of $\mathcal{H}_{E}$ with $D_{-\frac{1}{3}}^{+}$contains an indecomposable representation, one may 
expect that also the fusion of $\mathcal{H}_{E}$ with itself may be indecomposable. In order to test this further we have determined the quotient space (4.4), and we have found

$$
\left(\mathcal{H}_{E} \otimes \mathcal{H}_{E}\right)_{\mathrm{f}}^{(+1)}=\{(m, m): m \in \mathbb{Z} \ldots\} \bigoplus E
$$

where both the $J_{0}^{3}$ and $L_{0}$ eigenvalue of $(m, m)$ is $m$. This differs crucially from (5.13) in that now $m$ runs over all integers (rather than just the non-negative integers). Furthermore, we have determined the action of $J_{-1}^{+}$on these states - this is well-defined on the quotient space (7.1) - and we have found that, for some suitable normalisation,

$$
J_{-1}^{+}(m, m)=\frac{1}{3}(m+1)(3 m+1)(m+1, m+1) .
$$

In particular, it therefore follows that the state $(0,0)$ is not of the form $J_{-1}^{+}(-1,-1)$, in agreement with the result for the highest weight space. By symmetry, it also follows that the analogous quotient space where we exchange $J^{+}$and $J^{-}$has the same structure; in particular, we have states $(-m, m)$ for which

$$
J_{-1}^{-}(-m, m)=\frac{1}{3}(-m+1)(-3 m+1)(-m-1, m+1) .
$$

Summarising what we have found so far we therefore propose that

$$
\left(\mathcal{H}_{E} \otimes \mathcal{H}_{E}\right)_{\mathrm{f}}=\mathcal{R}_{0} \oplus \mathcal{H}_{E}
$$

where $\mathcal{R}_{0}$ is an extension of the vacuum representation.

The structure of the states $(-m, m)$ is the same as that of $\pi_{-1}\left(C_{\frac{2}{3}}\right)$, where $C_{\frac{2}{3}}$ is the other indecomposable representation of the zero mode algebra whose underlying vector space is the same as $C_{-\frac{1}{3}}$; its structure is schematically described by

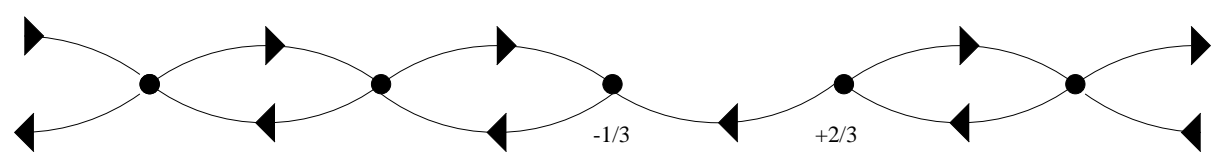

Fig. 3: The structure of $C_{\frac{2}{3}}$. The arrows describe the action of $J_{0}^{ \pm}$. 
Similarly, the states of the form $(m, m)$ have the same structure as $\pi_{1}\left(C_{-\frac{2}{3}}\right)$, where $C_{-\frac{2}{3}}$ is the 'mirror image' of $C_{\frac{2}{3}}$. Roughly speaking, the representation $\mathcal{R}_{0}$ is therefore the combination of these two representations. More precisely, we claim that $\mathcal{R}_{0}$ is generated by the action of the affine modes from $\omega$, where

$$
\begin{aligned}
J_{1}^{+} \omega & =(1,-1) \\
J_{1}^{-} \omega & =(-1,-1),
\end{aligned}
$$

and

$$
\begin{aligned}
J_{-1}^{-} J_{1}^{+} \omega & =\Omega=J_{-1}^{+} J_{1}^{-} \omega \\
J_{0}^{-} J_{0}^{+} \omega & =\gamma \Omega=J_{0}^{-} J_{0}^{+} \omega \\
L_{0} \omega & =\left(3+\frac{3}{2} \gamma\right) \Omega,
\end{aligned}
$$

where $\gamma \neq 0,-2$ is some constant. ${ }^{\star}$ The structure of this representation is schematically described by Figure 4 .

The fact that $J_{0}^{+} J_{0}^{-} \omega=J_{0}^{-} J_{0}^{+} \omega$ follows from the commutation relations of the affine algebra together with $J_{0}^{3} \omega=0$. If we postulate that $\omega$ is annihilated by

$$
\begin{aligned}
J_{n}^{a} \omega & =0 \quad n \geq 2 \\
J_{1}^{3} \omega & =0 \\
J_{1}^{+} J_{1}^{-} \omega & =0 \\
J_{1}^{\mp} J_{0}^{ \pm} \omega & =0,
\end{aligned}
$$

then the condition $J_{-1}^{-} J_{1}^{+} \omega=J_{-1}^{+} J_{1}^{-} \omega$ is again a consequence of $V_{1}(\mathcal{N}) \omega=0$. Since $\Omega$ is in the image of both $J_{-1}^{+}$and $J_{-1}^{-}$, it does not appear in the quotient space (4.4), in agreement with the result (5.13). We have therefore calculated the quotient space where we divide out the image of $J_{-n}^{3}, J_{-n-1}^{ \pm}$with $n \geq 1$, and we have found, that at $J_{0}^{3}$ charge zero, the relevant quotient space of the fusion product of $\mathcal{H}_{E}$ with itself is six-dimensional: three of these six states have conformal weight $-\frac{1}{3}, \frac{2}{3}$ and $\frac{5}{3}$, and correspond to states in $\mathcal{H}_{E}$. Of the remaining three states, one has conformal weight 2, while the other two form a Jordan block of length two at $h=0$ - these correspond to the states $\omega$ and $\Omega$. (In particular, this implies that $\gamma \neq-2$.) We have also checked that if we divide out further by the image of $J_{0}^{a}$, then the Jordan block disappears; this implies that $\Omega$ must be in the image of $J_{0}^{ \pm}$(and therefore that $\gamma \neq 0$ ).

* The representations that correspond to different values for $\gamma$ are inequivalent; the actual representation that occurs in the fusion product therefore has a specific value of $\gamma$. Unfortunately, this constant cannot be determined from the knowledge of the various quotient spaces that we have calculated. 


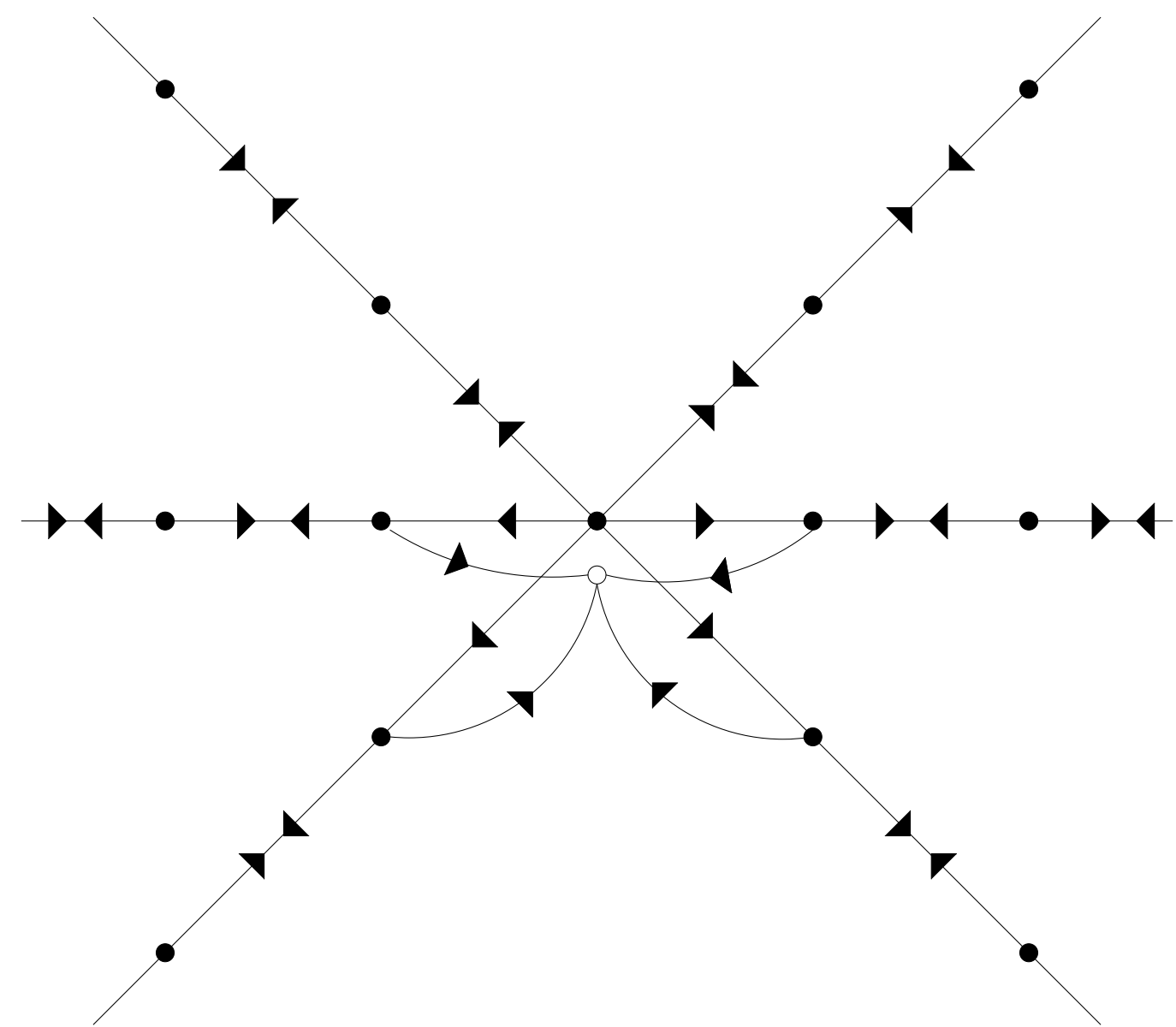

Fig. 4: The structure of the generating states of the representation $\mathcal{R}_{0}$. As before, the different states have been arranged according to their $J_{0}^{3}$ and $L_{0}$ charge. The two diagonal arrays of points represent the representations $\pi_{-1}\left(C_{\frac{2}{3}}\right)$ and $\pi_{1}\left(C_{-\frac{2}{3}}\right)$, while the horizontal line corresponds to the states that can be obtained by $J_{0}^{ \pm}$from $\omega$. The empty circle represents the state $\Omega$ whose position in the charge lattice has been slightly shifted so that it does not lie on top of the state $\omega$.

\section{Fusion closure}

Up to now we have described all fusion products involving the original representations in (3.7) as well as $\mathcal{H}_{E}$. However, as we have shown in the previous sections, the fusion rules still do not close on this set of representations since we generate the representations $\mathcal{R}_{-\frac{1}{3}}$ and $\mathcal{R}_{0}$ (as well as their images under $\pi_{s}$ ). Given that the structure of $\mathcal{R}_{-\frac{1}{3}}$ and $\mathcal{R}_{0}$ is quite complicated it would be very difficult to establish directly the fusion products involving these representations. However, we can use the fact that the fusion product is associative [19] to predict some other fusion products; furthermore, all fusion products can be determined once we have made a (fairly natural) assumption about one additional fusion product. 
First of all it follows from the associativity of the fusion product that

$$
\begin{aligned}
\left(D_{\frac{1}{3}}^{-} \otimes \mathcal{R}_{-\frac{1}{3}}\right)_{\mathrm{f}} & =\left[\left(D_{\frac{1}{3}}^{-} \otimes D_{-\frac{1}{3}}^{+}\right)_{\mathrm{f}} \otimes \mathcal{H}_{E}\right]_{\mathrm{f}} \\
& =\left[\left(\mathcal{H}_{0} \oplus \mathcal{H}_{E}\right)_{\mathrm{f}} \otimes \mathcal{H}_{E}\right]_{\mathrm{f}} \\
& =2 \mathcal{H}_{E} \oplus \mathcal{R}_{0} .
\end{aligned}
$$

In order to determine the remaining fusion products we now make the conjecture that

$$
\left(D_{-\frac{1}{3}}^{+} \otimes \mathcal{R}_{0}\right)_{\mathrm{f}}=\mathcal{R}_{-\frac{1}{3}}
$$

This is a very natural assumption since $\mathcal{R}_{0}$ is an extension of the vacuum representation, and one would therefore expect that the right-hand side of (8.2) is also an extension of $D_{-\frac{1}{3}}^{+}$; of the representations we have considered so far, the representation $\mathcal{R}_{-\frac{1}{3}}$ is the only representation with this property. We can also give some direct evidence for this conjecture by calculating the highest weight space of the fusion product in (8.2); this is done in appendix B.

With this assumption we can then determine the remaining fusion rules, using the associativity of the fusion product ${ }^{\dagger}$. For example, we have

$$
\left(\mathcal{R}_{-\frac{1}{3}} \otimes \mathcal{H}_{E}\right)_{\mathrm{f}}=\left[D_{-\frac{1}{3}}^{+} \otimes\left(\mathcal{H}_{E} \otimes \mathcal{H}_{E}\right)_{\mathrm{f}}\right]_{\mathrm{f}}=2 \mathcal{R}_{-\frac{1}{3}}
$$

We also find that

$$
\begin{aligned}
{\left[\mathcal{H}_{E} \otimes\left(D_{\frac{1}{3}}^{-} \otimes \mathcal{R}_{-\frac{1}{3}}\right)_{\mathrm{f}}\right]_{\mathrm{f}} } & =2\left(\mathcal{H}_{E} \otimes \mathcal{H}_{E}\right)_{\mathrm{f}} \bigoplus\left(\mathcal{H}_{E} \otimes \mathcal{R}_{0}\right)_{\mathrm{f}} \\
& =2 \mathcal{H}_{E} \oplus 2 \mathcal{R}_{0} \bigoplus\left(\mathcal{H}_{E} \otimes \mathcal{R}_{0}\right)_{\mathrm{f}}
\end{aligned}
$$

while on the other hand we have

$$
\begin{aligned}
{\left[\mathcal{H}_{E} \otimes\left(\mathcal{R}_{-\frac{1}{3}} \otimes D_{\frac{1}{3}}^{-}\right)_{\mathrm{f}}\right]_{\mathrm{f}} } & =\left[\left(\mathcal{H}_{E} \otimes \mathcal{R}_{-\frac{1}{3}}\right)_{\mathrm{f}} \otimes D_{\frac{1}{3}}^{-}\right]_{\mathrm{f}} \\
& =2\left(\mathcal{R}_{-\frac{1}{3}} \otimes D_{\frac{1}{3}}^{-}\right)_{\mathrm{f}} \\
& =4 \mathcal{H}_{E} \oplus 2 \mathcal{R}_{0},
\end{aligned}
$$

$\dagger$ As we shall see, the resulting 'fusion rules' are all non-negative integers; this is by no means guaranteed by our procedure, and therefore provides a consistency check on our assumption (8.2). In fact, if we had assumed that the right-hand-side of (8.2) was just $D_{-\frac{1}{3}}^{+}$the resulting 'fusion rules' would lead to some negative integers. 
from which we can conclude that

$$
\left(\mathcal{H}_{E} \otimes \mathcal{R}_{0}\right)_{\mathrm{f}}=2 \mathcal{H}_{E}
$$

Using similar techniques we also find that

$$
\begin{aligned}
\left(\mathcal{R}_{0} \otimes \mathcal{R}_{-\frac{1}{3}}\right)_{\mathrm{f}} & =2 \mathcal{R}_{-\frac{1}{3}} \\
\left(\mathcal{R}_{0} \otimes \mathcal{R}_{0}\right)_{\mathrm{f}} & =2 \mathcal{R}_{0} \\
\left(\mathcal{R}_{\frac{1}{3}} \otimes \mathcal{R}_{-\frac{1}{3}}\right)_{\mathrm{f}} & =2 \mathcal{R}_{0} \oplus 4 \mathcal{H}_{E} .
\end{aligned}
$$

Similar to what happened in [31] we observe that the fusion rules close among the representations $\mathcal{R}_{0}, \mathcal{R}_{\frac{1}{3}}$ and $\mathcal{H}_{E}$, together with their images under $\pi_{s}$. (In particular, this set also includes the representation $\mathcal{R}_{-\frac{1}{3}}=\pi_{-1}\left(\mathcal{R}_{\frac{1}{3}}\right)$.) In some sense this is again the natural set of representations to consider since $\mathcal{R}_{0}$ (and its images under $\pi_{\mp 1}$ ) contains $\mathcal{H}_{0}$ (and $D_{\mp \frac{2}{3}}^{ \pm}$) as a subrepresentation, and $\mathcal{R}_{ \pm \frac{1}{3}}$ contains $D_{ \pm \frac{1}{3}}^{\mp}$ as subrepresentations. Furthermore, these representations (together with their images under $\pi_{ \pm 1}$ ) contain all the different indecomposable representations of the zero mode algebra for which the Casimir takes value $C=-2 / 9$ and $J_{0}^{3}$ is in $\mathbb{Z} / 3$. Indeed there exist four such representations, namely $C_{ \pm \frac{1}{3}}$ and $C_{ \pm \frac{2}{3}}$; the former two are contained in $\mathcal{R}_{ \pm \frac{1}{3}}$, while the latter two arise in $\pi_{ \pm 1}\left(\mathcal{R}_{0}\right)$. It is therefore quite natural that the fusion rules should close on this set of representations.

Given that the fusion rules observe the symmetry (2.11), we can group the representations into orbits under $\pi_{s}^{\ddagger}$. Thus we have three orbits whose fusion closes among itself; the relevant fusion rules can then compactly be described by

$$
\begin{aligned}
{\left[\mathcal{R}_{0}\right] \otimes\left[\mathcal{R}_{0}\right] } & =2\left[\mathcal{R}_{0}\right] \\
{\left[\mathcal{R}_{0}\right] \otimes\left[\mathcal{H}_{E}\right] } & =2\left[\mathcal{H}_{E}\right] \\
{\left[\mathcal{R}_{0}\right] \otimes\left[\mathcal{R}_{\frac{1}{3}}\right] } & =2\left[\mathcal{R}_{\frac{1}{3}}\right] \\
{\left[\mathcal{H}_{E}\right] \otimes\left[\mathcal{H}_{E}\right] } & =\left[\mathcal{H}_{E}\right] \oplus\left[\mathcal{R}_{0}\right] \\
{\left[\mathcal{H}_{E}\right] \otimes\left[\mathcal{R}_{\frac{1}{3}}\right] } & =2\left[\mathcal{R}_{\frac{1}{3}}\right] \\
{\left[\mathcal{R}_{\frac{1}{3}}\right] \otimes\left[\mathcal{R}_{\frac{1}{3}}\right] } & =2\left[\mathcal{R}_{0}\right] \oplus 4\left[\mathcal{H}_{E}\right] .
\end{aligned}
$$

$\ddagger$ We do not propose that these orbits form representations of some loop group. We simply choose to combine these inequivalent representations (of the affine algebra) in order to obtain a compact formula for the fusion rules. The actual fusion rules (including the appropriate action of $\left.\pi_{s}\right)$ are described by the various formulae above. 
It is not difficult to check that the resulting fusion rules are associative and commutative; the $S$-matrix that diagonalises these fusion rules is then given by

$$
S=\left(\begin{array}{ccc}
\frac{1}{2 \sqrt{2}} & \frac{1}{2 \sqrt{2}} & \frac{1}{\sqrt{2}} \\
\frac{1}{\sqrt{2}} & \frac{1}{\sqrt{2}} & -\frac{1}{\sqrt{2}} \\
\frac{\sqrt{3}}{2 \sqrt{2}} & -\frac{\sqrt{3}}{2 \sqrt{2}} & 0
\end{array}\right)
$$

This $S$-matrix is not unitary let alone symmetric, and the fusion rules are therefore not 'self-dual' in the sense of Gannon [41]. Given that the $S$-matrix of Kac \& Wakimoto [7] is in general (i.e. for higher algebras) not unitary [42, the absence of unitary may not be surprising. The lack of symmetry may be related to the fact that the (unspecialised) character of the representation $\mathcal{H}_{E}$ vanishes for $0<z<1$. Unfortunately, since we do not know the characters of $\mathcal{R}_{0}$ and $\mathcal{R}_{\frac{1}{3}}$, we cannot check whether this $S$ matrix has an interpretation in terms of the modular transformation of these characters. Also, it is not clear whether it is appropriate to group these representations into the above orbits. For example, there are arguments why it may be natural to consider the orbits of $\pi_{2 s}$ or $\pi_{3 s}$ (where $s$ is again an integer) instead; on the other hand, this does not seem to improve the structural properties of the $S$-matrix: in either case the resulting $S$-matrix is not unitary nor symmetric.

Obviously, this $S$-matrix is not at all similar to the $S$-matrix obtained by Kac \& Wakimoto [7]. However, this is not surprising since the latter has an interpretation in terms of fusion rules that correspond to a different subset of representations (that also closes under fusion). As was pointed out by Ramgoolam [32], we have the (formal) character identity

$$
\chi_{D_{-\frac{1}{3}}^{+}}(\tau, z)=-\chi_{D_{\frac{2}{3}}^{-}}(\tau, z) .
$$

Thus, up to some trivial signs, the $S$-matrix of Kac \& Wakimoto equally describes the modular transformation rules of the three representations $\mathcal{H}_{0}, D_{\mp \frac{2}{3}}^{ \pm}$. Once these signs are appropriately introduced, the fusion rules that are associated to this modified $S$-matrix via the Verlinde formula [5] define non-negative integer fusion coefficients ${ }^{\star}$

$$
\begin{aligned}
{\left[D_{-\frac{2}{3}}^{+}\right] \otimes\left[D_{\frac{2}{3}}^{-}\right] } & =\left[\mathcal{H}_{0}\right] \\
{\left[D_{-\frac{2}{3}}^{+}\right] \otimes\left[D_{-\frac{2}{3}}^{+}\right] } & =\left[D_{\frac{2}{3}}^{-}\right] \\
{\left[D_{\frac{2}{3}}^{-}\right] \otimes\left[D_{\frac{2}{3}}^{-}\right] } & =\left[D_{-\frac{2}{3}}^{+}\right] .
\end{aligned}
$$

* The fusion rule coefficients that are obtained from the unmodified Kac-Wakimoto $S$-matrix are not non-negative integers as was first observed by [43] (see also [42]). 
From the point of view of the modular properties it is natural to group together representations that are related by $\pi_{3 s}$ since only then the conformal weight of the corresponding representations is guaranteed to differ by an integer (see (2.8) with $k=-4 / 3$ ). Actually more is true in the present case since it follows from the explicit formula for the characters [7] that

$$
\chi_{\pi_{s}\left(\mathcal{H}_{0}\right)}(\tau ; z)=\left\{\begin{array}{cl}
\chi_{\mathcal{H}_{0}}(\tau ; z) & \text { if } s=0 \bmod 3 \\
\chi_{D_{\frac{2}{3}}^{-}}(\tau ; z) & \text { if } s=1 \bmod 3 \\
\chi_{D_{-\frac{2}{3}}^{+}}(\tau ; z) & \text { if } s=-1 \bmod 3 .
\end{array}\right.
$$

Thus the equivalence classes in (8.11) correspond naturally to the images of the representations under $\pi_{3 s}$. These fusion rules then agree precisely with what we have claimed above; in fact, they are a direct consequence of (2.11) together with (3.11).

\section{Conclusions}

In this paper we have determined the fusion rules of $s u(2)$ at the fractional level $k=-4 / 3$. Starting with the 'admissible' highest weight representations we have shown that we generate representations that are not (conformal) highest weight representations. This is an immediate consequence of the automorphism symmetry (2.11) which we have confirmed in a number of cases directly. We have also found that some of the fusion products are not completely decomposable, and that they contain representations for which the action of $L_{0}$ is not diagonalisable. We have found a set of three representations (two of which are indecomposable) whose fusion closes among itself (together with its images under the action of the automorphism).

There are a few obvious directions in which the results of this paper should be extended. First of all, it would be important to understand the structure of the various indecomposable representations that we have found in more detail. This will presumably require a calculation of some even larger quotient spaces (that uncover more of the structure of the resulting representation). All of the calculations that we have done in this paper were in essence done by hand; in order to make further progress it is presumably necessary to implement these calculations on a computer.

It would also be interesting to understand the fusion of $s u(2)$ for the other admissible fractional levels ( $k=-4 / 3$ is only the simplest such example). Furthermore, it would be interesting to understand what happens for algebras of larger rank, such as su(3), etc. 
At any rate, the results of this paper suggest that the fusion of the admissible representations of all fractional level WZW models will contain indecomposable (and more specifically logarithmic) representations. This seems to indicate that, despite what one may have thought originally, 'logarithmic representations' do occur quite generically.

One of the original motivations for this work was the technical similarity between the representation theory for fractional level $s u(2)$ and for the WZW model that corresponds to the non-compact group $S U(1,1)$. The latter model is believed to describe string compactification on $A d S_{3}$, and therefore plays an important role in the analysis of the AdS/CFT correspondence [44,45, 46, 47, 48,49,50]. There are some indications that the fusion rules of the WZW model based on the group $S U(1,1)$ do indeed contain logarithmic representations [51,52,53,54,55]. It would be interesting to see whether the techniques used above can shed further light on this issue. It would also be interesting to study D-branes in these backgrounds. For the case of the local logarithmic theory constructed in [56] (see also [57]) the construction of boundary states has recently been accomplished in [58], following the programme outlined in [59]. It would be interesting to see whether an analogous construction can be performed for the fractional level WZW models, or indeed $S U(1,1)$.

\section{Acknowledgements}

I thank Alexander Ganchev, Terry Gannon, Andreas Recknagel, Mark Walton and Gérard Watts for useful discussions, and in particular Alexander Ganchev and Terry Gannon for much needed encouragement.

I am grateful to the Royal Society for a University Research Fellowship.

\section{Appendix A. The fusion calculation for $\mathcal{R}_{-\frac{1}{3}}$}

In this appendix we want to give some of the details of the derivation of our central result (6.7) and (6.8). First we want to describe the space

$$
\left(D_{-\frac{1}{3}}^{+} \otimes \mathcal{H}_{E}\right)_{\mathrm{f}}^{(+1)}
$$

Because of the null vector $\mathcal{N}_{1}$, we can rewrite any state of the form $\left(J_{-1}^{+}\right)^{l}|m\rangle$ in terms of states that do not involve any $J_{-1}^{+}$modes; thus we can apply the relations in (4.2) to show 
that (A.1) is spanned by states in the tensor product of the two highest weight spaces. Next we want to derive further restrictions by using the null vectors of the two representations. It is most convenient to consider a null-vector (at grade two) that does not involve any $J_{-1}^{+}$ modes (since these cannot easily be 'removed' using the algorithm). By taking a suitable combination of descendants of $\mathcal{N}_{1}$ and $\mathcal{N}_{2}$ we find that the representation $D_{-\frac{1}{3}}^{+}$has the null vector

$$
\begin{aligned}
& \mathcal{N}_{-\frac{1}{3}}=\left(9 J_{-1}^{3} J_{-1}^{3} J_{0}^{-} J_{0}^{-}-45 J_{-2}^{3} J_{0}^{-} J_{0}^{-}+14 J_{-1}^{-} J_{-1}^{-}+36 J_{-1}^{-} J_{-1}^{3} J_{0}^{-}\right. \\
& \left.-48 J_{-2}^{-} J_{0}^{-}+9 J_{-2}^{+} J_{0}^{-} J_{0}^{-} J_{0}^{-}\right)\left|-\frac{1}{3}\right\rangle \text {. }
\end{aligned}
$$

Using the algorithm (4.2) we then obtain (after some algebra) the relation

$$
\begin{array}{r}
0=\left[-\frac{14}{81}(3 j+2)(3 j+1)(3 j-1)(3 j-2)(\mathbb{1} \otimes \mathbb{1})+\frac{4}{3}(3 j+4)(3 j+2)(3 j+1)\left(J_{0}^{-} \otimes J_{0}^{+}\right)\right. \\
\left.\quad-9(j+1)(j+6)\left(J_{0}^{-} J_{0}^{-} \otimes J_{0}^{+} J_{0}^{+}\right)+9\left(J_{0}^{-} J_{0}^{-} J_{0}^{-} \otimes J_{0}^{+} J_{0}^{+} J_{0}^{+}\right)\right]\left|-\frac{1}{3}\right\rangle \otimes|j-1\rangle .
\end{array}
$$

Similarly, the representation $\mathcal{H}_{E}$ has a null vector at grade two that does not involve $J_{-1}^{+}$; it is given by

$$
\begin{aligned}
\mathcal{N}_{E}=(3(3 j & +1)(3 j+2)(3 j+4) J_{-1}^{3} J_{-1}^{3}+(3 j+1)(3 j+2)(3 j+4)(6 j-1) J_{-2}^{3} \\
& +81 j J_{-1}^{-} J_{-1}^{-} J_{0}^{+} J_{0}^{+}+3(3 j+4)\left(9 j^{2}-3 j-8\right) J_{-2}^{-} J_{0}^{+} \\
& \left.+18(3 j+1)(3 j+4) J_{-1}^{-} J_{-1}^{3} J_{0}^{+}+3(3 j+1)(3 j+2)(3 j+4) J_{-2}^{+} J_{0}^{-}\right)|j\rangle .
\end{aligned}
$$

Considering $0=\left|-\frac{4}{3}\right\rangle \otimes \mathcal{N}_{E}$, and using the algorithm (4.2) we then obtain (again after some algebra) the relation

$$
\begin{aligned}
0=\left[-\frac{2}{9}\right. & (3 j+1)(3 j+2)(3 j+4)(3 j-1)(3 j-2)(\mathbb{1} \otimes \mathbb{1}) \\
& +4(3 j+1)(3 j+2)(3 j+4)(2 j+1)\left(J_{0}^{-} \otimes J_{0}^{+}\right) \\
& -9(3 j+4)\left(3 j^{2}+13 j+2\right)\left(J_{0}^{-} J_{0}^{-} \otimes J_{0}^{+} J_{0}^{+}\right) \\
& \left.+81 j\left(J_{0}^{-} J_{0}^{-} J_{0}^{-} \otimes J_{0}^{+} J_{0}^{+} J_{0}^{+}\right)\right]\left|-\frac{1}{3}\right\rangle \otimes|j-1\rangle .
\end{aligned}
$$

Remarkably, the two equations (A.3) and (A.5) are linearly independent except for $j=1$. For $j \neq 1$, we can therefore combine these two equations to obtain

$$
\begin{gathered}
0=\left[\frac{8}{9}(3 j+1)(3 j-1)(3 j-2)(\mathbb{1} \otimes \mathbb{1})-4(3 j+1)(3 j+4)\left(J_{0}^{-} \otimes J_{0}^{+}\right)\right. \\
\left.+36\left(J_{0}^{-} J_{0}^{-} \otimes J_{0}^{+} J_{0}^{+}\right)\right]\left|-\frac{1}{3}\right\rangle \otimes|j-1\rangle .
\end{gathered}
$$


On the two-dimensional space spanned by $\left|-\frac{1}{3}\right\rangle \otimes|j-1\rangle$ and $\left(J_{0}^{-} \otimes J_{0}^{+}\right)\left|-\frac{1}{3}\right\rangle \otimes|j-1\rangle$ we have determined the action of $L_{0}$ using the comultiplication formula

$$
\Delta\left(L_{0}\right)=\left(L_{-1} \otimes \mathbb{1}\right)+\left(L_{0} \otimes \mathbb{1}\right)+\left(\mathbb{1} \otimes L_{0}\right)
$$

and the Sugawara expression for $L_{-1}$. Using (A.6) to rewrite $\left(J_{0}^{-} J_{0}^{-} \otimes J_{0}^{+} J_{0}^{+}\right)\left|-\frac{1}{3}\right\rangle \otimes|j-1\rangle$ in terms of the above two states, we have found that the action of $L_{0}$ is described by the matrix

$$
L_{0}=\left(\begin{array}{cc}
-1 & -\frac{2}{27}(3 j-1)(3 j-2) \\
\frac{1}{\left(j-\frac{2}{3}\right)} & \left(j-\frac{2}{3}\right)
\end{array}\right) .
$$

This matrix has eigenvalues $-\frac{1}{3}$ and $j-\frac{4}{3}$, as claimed.

On the other hand, for $J_{0}^{3}=-\frac{1}{3}$, the relevant space is three-dimensional, and can be taken to be spanned by $\left|-\frac{1}{3}\right\rangle \otimes|0\rangle,\left|-\frac{4}{3}\right\rangle \otimes|1\rangle$ and $\left|-\frac{7}{3}\right\rangle \otimes|2\rangle$. Using (A.3) (or (A.5)) we then find that the action of $L_{0}$ is described by the matrix

$$
L_{0}=\left(\begin{array}{rrc}
-\frac{5}{3} & -\frac{4}{27} & 0 \\
\frac{27}{2} & \frac{1}{3} & -\frac{100}{27} \\
-\frac{27}{8} & 0 & \frac{4}{3}
\end{array}\right) .
$$

This matrix is then conjugate to the Jordan normal form matrix

$$
L_{0}=\left(\begin{array}{ccc}
\frac{2}{3} & 0 & 0 \\
0 & -\frac{1}{3} & 1 \\
0 & 0 & -\frac{1}{3}
\end{array}\right)
$$

The two states that span the Jordan block with $h=-\frac{1}{3}$ are explicitly given by

$$
\begin{aligned}
& \left(-\frac{1}{3},-\frac{1}{3}\right)_{1}=\frac{11}{9}\left(\left|-\frac{1}{3}\right\rangle \otimes|0\rangle\right)-\frac{7}{2}\left(\left|-\frac{4}{3}\right\rangle \otimes|1\rangle\right)+\frac{9}{8}\left(\left|-\frac{7}{3}\right\rangle \otimes|2\rangle\right) \\
& \left(-\frac{1}{3},-\frac{1}{3}\right)_{2}=-\frac{10}{9}\left(\left|-\frac{1}{3}\right\rangle \otimes|0\rangle\right)+10\left(\left|-\frac{4}{3}\right\rangle \otimes|1\rangle\right)-\frac{9}{4}\left(\left|-\frac{7}{3}\right\rangle \otimes|2\rangle\right) .
\end{aligned}
$$

When we quotient further to obtain the 'highest weight space' we have the additional relations

$$
\begin{aligned}
& 0=-\left(\left|-\frac{1}{3}\right\rangle \otimes|0\rangle\right)+\frac{9}{2}\left(\left|-\frac{4}{3}\right\rangle \otimes|1\rangle\right) \\
& 0=\frac{2}{9}\left(\left|-\frac{1}{3}\right\rangle \otimes|0\rangle\right)-3\left(\left|-\frac{4}{3}\right\rangle \otimes|1\rangle\right)+\frac{9}{10}\left(\left|-\frac{7}{3}\right\rangle \otimes|2\rangle\right) .
\end{aligned}
$$

Using the relations in (A.12) it is then easy to see that $\left(-\frac{1}{3},-\frac{1}{3}\right)_{2} \cong 0$ in the highest weight space. 


\section{Appendix B. The highest weight space of $\left(D_{-\frac{1}{3}}^{+} \otimes \mathcal{R}_{0}\right)$}

In this appendix we want to determine the highest weight space of the fusion product in (8.2). Using (4.2) it is easy to see that this highest weight space can be taken to be contained in $\left(D_{-\frac{1}{3}}^{+}\right)^{(0)} \otimes \mathcal{R}_{0}$. Naively, one may further think that one can also restrict $\mathcal{R}_{0}$ to its highest weight space; however, this is not quite correct since the $L_{0}$ spectrum of $\mathcal{R}_{0}$ is unbounded from below. However, we can derive the recursion relations

$$
\begin{aligned}
\left(J_{0}^{+}|j\rangle \otimes(m, m)\right) & \propto(|j\rangle \otimes(m+1, m+1)) \\
\left(J_{0}^{+}|j\rangle \otimes \omega\right) & \propto(|j\rangle \otimes(1,1)) \\
\left(J_{0}^{-}|j\rangle \otimes(-m, m)\right) & \propto(|j\rangle \otimes(-m-1, m+1)) \\
\left(J_{0}^{-}|j\rangle \otimes \omega\right) & \propto(|j\rangle \otimes(-1,1)),
\end{aligned}
$$

where $m \neq 0,|j\rangle \in\left(D_{-\frac{1}{3}}^{+}\right)^{(0)}$, and we have used the same notation as in section 7 as well as $\Omega=(0,0)$; none of the proportionality constants vanishes. If $m \geq 1$, these relations imply that

$$
\begin{aligned}
(|j\rangle \otimes(m, m)) & \propto(|j+m\rangle \otimes \omega) \\
(|j\rangle \otimes(-m, m)) & \propto(|j-m\rangle \otimes \omega),
\end{aligned}
$$

where the proportionality constants are non-zero, and $|l\rangle=0$ if $l>-\frac{1}{3}$. These states have the same $J_{0}^{3}$ spectrum as $\left(D_{-\frac{1}{3}}^{+}\right)^{(0)}$.

It remains to analyse the states with $m \leq 0$. First of all, it follows from (B.1) together with the fact that $\left|-\frac{1}{3}\right\rangle$ is annihilated by $J_{0}^{+}$that

$$
(|j\rangle \otimes(m, m)) \propto 0 \quad \text { for } m \leq 0 .
$$

In particular, this implies that $|j\rangle \otimes \Omega \cong 0$ for all $j$. It then also follows from (B.1) that

$$
|j\rangle \otimes(-m, m) \cong 0 \quad \text { if } j-m \leq-\frac{1}{3}
$$

since we can use $(\mathbb{B . 1})$ repeatedly to rewrite $|j\rangle \otimes(-m, m)$ in terms of $|j-m\rangle \otimes \Omega \cong 0$. However, if $j-m \geq \frac{2}{3}$, this argument breaks down and we can only conclude that

$$
(|j\rangle \otimes(-m, m)) \propto\left(\left|-\frac{1}{3}\right\rangle \otimes\left(j-m+\frac{1}{3},-j+m-\frac{1}{3}\right)\right) .
$$

Thus we get one additional state for each $J_{0}^{3}$ eigenvalue of $\frac{2}{3}+n$ where $n \geq 0$; these combine with the states in (B.2) to give the complete highest weight space of $\mathcal{R}_{-\frac{1}{3}}$. 


\section{References}

[1] E. Witten, Non-abelian bosonization in two dimensions, Commun. Math. Phys. 92, 455 (1984).

[2] D. Gepner, E. Witten, String theory on group manifolds, Nucl. Phys. B278, 493 (1986).

[3] V.G. Kac, Infinite-dimensional Lie algebras, Cambridge (1990).

[4] M.A. Walton, Fusion rules in Wess-Zumino-Witten models, Nucl. Phys. B340, 777 (1990).

[5] E. Verlinde, Fusion rules and modular transformations in 2D conformal field theory, Nucl. Phys. B300 [FS22], 360 (1988).

[6] M.R. Gaberdiel, P. Goddard, Axiomatic conformal field theory, Commun. Math. Phys. 209, 549 (2000); hep-th/9810019.

[7] V.G. Kac, M. Wakimoto, Modular invariant representations of infinite dimensional Lie algebras and superalgebras, Proc. Natl. Acad. Sci. USA 85, 4956 (1988).

[8] D. Bernard, G. Felder, Fock representations and BRST cohomology in sl(2) current algebra, Commun. Math. Phys. 127, 145 (1990).

[9] S. Panda, Fractional level current algebras and spectrum of $C<1$ minimal models coupled to gravity, Phys. Lett. B251, 61 (1990).

[10] H. Awata, Y. Yamada, Fusion rules for the fractional level $\widehat{\operatorname{sl}(2)}$ algebra, Mod. Phys. Lett. A7, 1185 (1992).

[11] V.S. Dotsenko, Solving the su(2) conformal field theory with the Wakimoto free field representation, Nucl. Phys. B358, 547 (1991).

[12] B. Feigin, F. Malikov, Fusion algebra at a rational level and cohomology of nilpotent subalgebras of supersymmetric sl(2), Lett. Math. Phys. 31, 315 (1994); hepth/9310004.

[13] O. Andreev, Operator algebra of the SL(2) conformal field theories, Phys. Lett. B363, 166 (1995); hep-th/9504082.

[14] C. Dong, H. Li, G. Mason, Vertex operator algebras associated to admissible representations of $\hat{s l} l_{2}$, Commun. Math. Phys. 184, 65 (1997); q-alg/9509026.

[15] J.L. Petersen, J. Rasmussen, M. Yu, Fusion, crossing and monodromy in conformal field theory based on sl(2) current algebra with fractional level, Nucl. Phys. B481, 577 (1996); hep-th/9607129.

[16] P. Furlan, A.Ch. Ganchev, V.B. Petkova, $A_{1}^{(1)}$ admissible representations - fusion transformations and local correlators, Nucl. Phys. B491, 635 (1997); hep-th/9608018.

[17] A.Ch. Ganchev, V.B. Petkova, G.M.T. Watts, A note on decoupling conditions for generic level $\hat{s l}(3)_{k}$ and fusion rules, hep-th/9906139.

[18] G. Moore, N. Seiberg, Classical and quantum conformal field theory, Commun. Math. Phys. 123, 177 (1989). 
[19] M.R. Gaberdiel, Fusion in conformal field theory as the tensor product of the symmetry algebra, Int. Journ. Mod. Phys. A9, 4619 (1994); hep-th/9307183.

[20] M.R. Gaberdiel, Fusion rules of chiral algebras, Nucl. Phys. B417, 130 (1994); hepth/9309105.

[21] W. Nahm, Quasi-rational fusion products, Int. Journ. Mod. Phys. B8, 3693 (1994); hep-th/9402039.

[22] M.R. Gaberdiel, H.G. Kausch, Indecomposable fusion products, Nucl. Phys. B477, 293 (1996); hep-th/9604026.

[23] V. Gurarie, Logarithmic operators in conformal field theory, Nucl. Phys. B410, 535 (1993); hep-th/9303160.

[24] M.A. Flohr, On modular invariant partition functions of conformal field theories with logarithmic operators, Int. J. Mod. Phys. A11, 4147 (1996); hep-th/9509166.

[25] M. Flohr, On fusion rules in logarithmic conformal field theories, Int. J. Mod. Phys. A12, 1943 (1997); hep-th/9605151.

[26] M.R. Rahimi Tabar, A. Aghamohammadi, M. Khorrami, The logarithmic conformal field theories, Nucl. Phys. B497, 555 (1997); hep-th/9610168.

[27] F. Rohsiepe, On reducible but indecomposable representations of the Virasoro algebra, hep-th/9611160.

[28] M. Flohr, Singular vectors in logarithmic conformal field theories, Nucl. Phys. B514, 523 (1998); hep-th/9707090.

[29] J.-S. Caux, I.I. Kogan, A.M. Tsvelik, Logarithmic operators and hidden continuous symmetry in critical disordered models, Nucl. Phys. B466, 444 (1996); hepth/9511134.

[30] J.-S. Caux, I.I. Kogan, A. Lewis, A.M. Tsvelik, Logarithmic operators and dynamical extention of the symmetry group in the bosonic $S U(2)_{0}$ and $S U S Y S U(2)_{2} W Z N W$ models, Nucl. Phys. B489, 469 (1997); hep-th/9606138.

[31] M.R. Gaberdiel, H.G. Kausch, A rational logarithmic conformal field theory, Phys. Lett. B386, 131 (1996); hep-th/9606050.

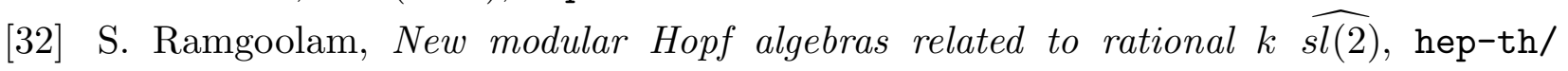
9301121.

[33] A. Pressley, G.B. Segal, Loop groups, Oxford University Press, Oxford (1986).

[34] M.R. Gaberdiel, WZW models of general simple groups, Nucl. Phys. B460, 181 (1996); hep-th/9508105.

[35] M.R. Gaberdiel, Fusion of twisted representations, Int. Journ. Mod. Phys. A12, 5183 (1997); hep-th/9607036.

[36] Y. Zhu, Modular invariance of characters of vertex operator algebras, J. Amer. Math. Soc. 9, 237 (1996). 
[37] W. Eholzer, M. Flohr, A. Honecker, R. Hübel, W. Nahm, R. Varnhagen, Representations of $W$-algebras with two generators and new rational models, Nucl. Phys. B383, 249 (1992).

[38] I. Frenkel, J. Lepowsky, A. Meurman, Vertex Operator Algebras and the Monster, Academic Press (1988).

[39] C. Dong, H. Li, G. Mason, Twisted representations of vertex operator algebras, Math. Ann. 310, 571 (1998); q-alg/9509005.

[40] W. Eholzer, M.R. Gaberdiel, Unitarity of rational $N=2$ superconformal theories, Commun. Math. Phys. 186, 61 (1997); hep-th/9601163.

[41] T. Gannon, Modular data: the algebraic combinatorics of conformal field theory, math.QA/0103044.

[42] P. Mathieu, M.A. Walton, Fractional level Kac-Moody algebras and nonunitary coset conformal field theories, Prog. Theor. Phys. Suppl. 102, 229 (1990).

[43] I.G. Koh, P. Sorba, Fusion rules and (sub)-modular invariant partition functions in non-unitary theories, Phys. Lett. B215, 723 (1988).

[44] J. Maldacena, The large N limit of superconformal field theories and supergravity, Adv. Theor. Math. Phys. 2, 231 (1998); hep-th/9711200.

[45] J.M. Evans, M.R. Gaberdiel, M.J. Perry, The No-ghost theorem for AdS $S_{3}$ and the stringy exclusion principle, Nucl. Phys. B535, 152 (1998); hep-th/9806024.

[46] J. de Boer, Six-Dimensional supergravity on $S^{3} \times A d S_{3}$ and $2 d$ conformal field theory, Nucl. Phys. B548, 139 (1999); hep-th/9806104.

[47] A. Giveon, D. Kutasov, N. Seiberg, Comments on string theory on $A d S_{3}$, Adv. Theor. Math. Phys. 2, 733 (1998); hep-th/9806194.

[48] J. Teschner, Operator product expansion and factorization in the $\mathrm{H}_{3}^{+}$-WZNW model, Nucl. Phys. B571, 555 (2000); hep-th/9906215.

[49] J. Maldacena, H. Ooguri, Strings in $A d S_{3}$ and the $S L(2, R)$ WZW model. Part 1: The spectrum, Int. J. Mod. Phys. A16, 677 (2001); hep-th/0001053.

[50] J. Maldacena, H. Ooguri, J. Son, Strings in $A d S_{3}$ and the $S L(2, R)$ WZW model. Part 2: Euclidean black hole, hep-th/0005183.

[51] A. Bilal, I.I. Kogan, On gravitational dressing of $2 D$ field theories in chiral gauge, Nucl. Phys. B449, 569 (1995); hep-th/9503209.

[52] I.I. Kogan, A. Lewis, O.A. Soloviev, Knizhnik-Zamolodchikov-type equations for gauged WZNW models, Int. J. Mod. Phys. A13, 1345 (1998); hep-th/9703028.

[53] I.I. Kogan, A.M. Tsvelik, Logarithmic operators in the theory of plateau transition, Mod. Phys. Lett. A15, 931 (2000); hep-th/9912143.

[54] A. Nichols, Sanjay, Logarithmic operators in the $S L(2, R)$ WZNW model, Nucl. Phys. B597, 633 (2001); hep-th/0007007.

[55] A. Lewis, Logarithmic CFT on the boundary and the world-sheet, hep-th/0009096. 
[56] M.R. Gaberdiel, H.G. Kausch, A local logarithmic conformal field theory, Nucl. Phys. B538, 631 (1999); hep-th/9807091.

[57] H.G. Kausch, Symplectic Fermions, Nucl. Phys. B583, 513 (2000); hep-th/0003029.

[58] S. Kawai, J.F. Wheater, Modular transformation and boundary states in logarithmic conformal field theory, hep-th/0103197.

[59] I.I. Kogan, J.F. Wheater, Boundary logarithmic conformal field theory, Phys. Lett. B486, 353 (2000); hep-th/0003184. 